\title{
Isotropic-nematic transition of $D$-dimensional hard convex bodies within the effective-liquid approach
}

\author{
José A. Cuesta and Carlos F. Tejero \\ Facultad de Ciencias Físicas, Universidad Complutense de Madrid, 28040 Madrid, Spain
}

\author{
Marc Baus \\ Physique Statistique et Plasmas, Case Postale 231, Université Libre de Bruxelles, B-1050 Bruxelles, Belgium
}

(Received 29 October 1991; revised manuscript received 30 December 1991)

\begin{abstract}
Density-functional theory within the effective-liquid approximation is applied to the problem of the isotropic-nematic transition of $D$-dimensional hard convex bodies. It is shown that the free-energy functional factorizes into its radial and angular contributions. Due to this factorization two different versions of the self-consistent equations can be implemented, and it is shown that in $D=3$ they coincide with previous theories. In the present work all the formulas are worked out with a particular choice for the angular distribution: the one-order-parameter approximation. The problem of determining the excluded volume of two hard convex bodies is discussed. For hard ellipsoids the Gaussian-overlap approximation is used, whereas an exact formula is given for the excluded volume of two hard spherocylinders. For $D=2$ the virial coefficients of the isotropic phase as well as the transition are incorrectly predicted, due to the approximation of the direct correlation function involved. For $D=3$ the results are in very good agreement with simulations. Expression and data for the isotropic-nematic transition for $D>3$ are also provided. Extensive comparisons with the results of other theories are made throughout. The oneorder-parameter approximation is proven not to alter the order of the transition. Finally, it is shown that the present approximation becomes exact in the large- $D$ limit.
\end{abstract}

PACS number(s): 61.30.By, 64.70.Md, 05.70.Fh

\section{INTRODUCTION}

Freezing can be considered as the paradigm of a firstorder phase transition. Although in the last decades we have gained a great deal of understanding, we still lack a real first-principles theory that accounts for the mechanism of freezing. An important breakthrough was attained when Alder and Wainwright [1] proved, by molecular-dynamics (MD) simulations, that a system of hard spheres (HS's) undergoes a first-order phase transition from a disordered fluid phase to an ordered solid in which spheres occupy the sites of a three-dimensional (3D) lattice. The important idea underlying this result is the fact that repulsive forces alone can induce a solid phase, contrary to the then-widespread idea that attractive forces are needed. The later discovery of a 2D solid in a system of hard disks [2] confirmed this point. Since then all theoretical efforts have been devoted to reproducing the thermodynamics and structure of such purely repulsive systems. Along this line of work the most remarkable success has been achieved via densityfunctional theory [3] (DFT). The idea underlying DFT is that the thermodynamic potentials of a system can be written as functionals of the one-body density function [4]. The main advantage of this theory, as already proven by the pioneering work of Ramakrishnan and Yussouf [5], is to be capable of obtaining insight into the thermodynamics of the solid using only the well-known structure of the fluid phase [6] as input. Among the different approaches that have been developed in the context of DFT [3], the effective-liquid approximation (ELA) for the description of the freezing of HS's [7] has obtained very good agreement with computer simulations.

The study of liquid-crystalline mesophases has paralleled that of freezing. By liquid crystals (LC's) we refer to those phases that exhibit intermediate order between the crystalline order of solids and the full disorder of fluids [8]. It is well known that some anisotropy in the basic components is necessary in order to obtain these mesophases; once this anisotropy is present the kind of order we can find is either orientational order or partial positional order or a combination of both. The early work by Onsager [9] on the transition from an isotropic (I) fluid to a nematic ( $N$ ) (orientationally ordered) fluid of infinitely long hard rods suggested, as in the case of HS freezing, that purely repulsive forces can account for the existence of mesophases. The later results obtained in computer simulations [10-18] and theoretical studies [19-30] have confirmed this idea. Nowadays it is widely accepted that all mesophases found in nature (and even others one can imagine by symmetry arguments) can be obtained from a proper choice of a hard-molecule model. As in the case of HS freezing, the best theoretical frame for the description of hard-core models of LC's has been DFT [19-30].

Concerning the $I-N$ transition, the ELA provides again a fairly accurate description [27-29]. Besides, it is a very general and flexible scheme, so that many other theoretical approaches can be reformulated in terms of the ELA, as was already shown in a previous work [29]. It is the purpose of the present paper to report a detailed study of the $I-N$ transition of $D$-dimensional hard convex bodies 
(HCB's). To this end, we develop in Sec. II the formulas for the thermodynamics of HCB's, starting from the general expressions of the DFT and working them out within the ELA. In Sec. III we approximate further the expressions by using a one-parameter trial function for the angular distribution, transforming what was previously a functional into a simple function of the corresponding order parameter. These expressions contain the volume excluded to a body due to the presence of another one, when their orientations are kept fixed. In Sec. IV we discuss this "excluded volume" for two important molecular shapes: hard ellipsoids (HE's) and hard spherocylinders (HSC's). Section V is devoted to the results for the isotropic phases and the comparison with other theories and computer simulations. Special emphasis is put on the virial coefficients (mainly for the $2 \mathrm{D}$ system), because their features reveal much about the approximations that are being used. The nematic phase as well as the $I-N$ transition for HE's is studied in Sec. VI. In Sec. VII we discuss the validity of the results obtained for the transition when compared to those obtained from the exact Euler-Lagrange equation for the angular distribution. Section VIII is devoted to an analysis of the limit of infinitely large eccentricity and its relation to the system of HSC's. Finally, the large dimensionality limit of the ELA is obtained in Sec. IX and proven to coincide with the exact results.

\section{THERMODYNAMICS OF D-DIMENSIONAL HARD CONVEX BODIES}

We consider a system of $N$ uniaxial hard convex bodies enclosed in a $D$-dimensional volume $V$. Let $\rho=N / V$ and $\phi=F / \rho V$ denote, respectively, the average density and the free energy $(F)$ per particle of such a system. According to the DFT [4] the free energy $\phi=\phi[\rho]$ is uniquely defined as a functional of the one-body density function $\rho(\mathbf{x})$, where $\mathbf{x}=(\mathbf{r}, \mathbf{u})$ denotes both the translational $(\mathbf{r})$ and orientational ( $\mathbf{u}$ ) degrees of freedom. This functional can be decomposed into a sum of three terms: the idealgas contribution $\phi_{\mathrm{id}}$ arising from the kinetic part of the Hamiltonian; the external-field contribution $\phi_{\text {ext }}$, and an excess term $\phi_{\text {ex }}$, due to the interactions between the particles of the system. Then $\phi=\phi_{\text {id }}+\phi_{\text {ext }}+\phi_{\text {ex }}$, with

$$
\begin{aligned}
\beta \phi_{\mathrm{id}}[\rho] & =\frac{1}{\rho V} \int d \mathbf{x} \rho(\mathbf{x})\{\ln [\Lambda \rho(\mathbf{x})]-1\} \\
\beta \phi_{\mathrm{ext}}[\rho] & =\frac{1}{\rho V} \int d \mathbf{x} \rho(\mathbf{x}) u(\mathbf{x}) \\
\beta \phi_{\mathrm{ex}}[\rho] & =-\frac{1}{\rho V} \int d \mathbf{x} \int d \mathbf{x}^{\prime} \rho(\mathbf{x}) \rho\left(\mathbf{x}^{\prime}\right) \\
& \times \int_{0}^{1} d \lambda(1-\lambda) c\left(\mathbf{x}, \mathbf{x}^{\prime} ;[\lambda \rho]\right)
\end{aligned}
$$

where $u(\mathbf{x})$ is the external potential acting on a particle of coordinates $\mathbf{x} ; \beta=1 / k_{B} T$ is the inverse temperature, and $\Lambda$ is the thermal de Broglie wavelength to the power
$D$. The normalization is chosen in such a way that $\int d \mathbf{r}=V$ and $\int d \mathbf{u}=1$. The function $c\left(\mathbf{x}, \mathbf{x}^{\prime} ;[\rho]\right)$ appearing in (2.3) is the direct correlation function (DCF) introduced by Ornstein and Zernike [31], which for a nonuniform phase is a functional of $\rho(\mathbf{x})$. The external field $u(\mathbf{x})$ is necessary in order to confine the system to a region of volume $V$ and to induce an orientational symmetry breaking in the case of the $N$ phase. Equivalently, we can disregard $\phi_{\text {ext }}$ by imposing the appropriate (boundary) conditions directly on $\rho(\mathbf{x})$. So, if we are only interested in the $I-N$ transition we can put $\rho(\mathbf{x})=\rho h(\mathbf{u})$ with $\int d \mathbf{u} h(\mathbf{u})=1$ since only orientational order will be present; $h(\mathbf{u})$ is then the orientational distribution function [32], i.e., $h(\mathbf{u}) d \mathbf{u}$ represents the probability for the orientation of a given molecule to be in a region $d \mathbf{u}$ around the vector $\mathbf{u}$. If we introduce this particular form of $\rho(\mathbf{x})$ in Eqs. (2.1) and (2.3) we get

$$
\begin{aligned}
& \beta \phi_{\mathrm{id}}[h]=\ln (\Lambda \rho)-1+\int d \mathbf{u} h(\mathbf{u}) \ln h(\mathbf{u}) \\
& \beta \phi_{\mathrm{ex}}[h]=-\rho \int d \mathbf{u} \int d \mathbf{u}^{\prime} h(\mathbf{u}) h\left(\mathbf{u}^{\prime}\right) \\
& \times \int_{0}^{1} d \lambda(1-\lambda) \\
& \times \int d \mathbf{r} c\left(\mathbf{r} ; \mathbf{u}, \mathbf{u}^{\prime} ;[\lambda h]\right)
\end{aligned}
$$

where the translational invariance of both phases ( $I$ and $N)$ has allowed us to write $c\left(\mathbf{x}, \mathbf{x}^{\prime} ;[\rho]\right)$ $=c\left(\mathbf{r}-\mathbf{r}^{\prime} ; \mathbf{u}, \mathbf{u}^{\prime} ;[h]\right)$.

At this point we face the problem of finding the expression of the DCF of an inhomogeneous phase. This has turned out to be a very difficult problem which has as yet only been solved exactly for some 1D systems [33]. For higher-dimensional systems one has to find a reasonable approximation for the DCF. To this purpose different strategies have been developed [21-24]. One of these circumvents the inhomogeneous DCF problem by translating the ELA formalism developed within the DFT of freezing [3] into the $I-N$ language, as done by Baus et al. [27] for 3D and by Cuesta, Tejero, and Baus [28] for 2D. The ELA prescription [7] consists of replacing the exact DCF of Eq. (2.5) by the DCF of the isotropic fluid $\left(c_{I}\right)$ but evaluated at an effective density $\bar{\rho}$ :

$$
c\left(\mathbf{r} ; \mathbf{u}, \mathbf{u}^{\prime} ;[h]\right)=c_{I}\left(\mathbf{r} ; \mathbf{u}, \mathbf{u}^{\prime} ; \bar{\rho}[h]\right) .
$$

As indicated in Eq. (2.6), $\bar{\rho}$ is, in general, a functional of $\rho h(\mathbf{u})$, i.e., $\bar{\rho}=\bar{\rho}[h]$. For the right-hand side of (2.6) we then use Pynn's approximation [34]; thus, if $\sigma\left(\hat{\mathbf{r}} ; \mathbf{u}, \mathbf{u}^{\prime}\right)$ denotes the contact distance of two HCB's of given orientations $\mathbf{u}, \mathbf{u}^{\prime}$ and center-to-center orientation $\widehat{\mathbf{r}} \equiv \mathbf{r} / r$, we will use as DCF for the HCB the expression

$$
c_{I}^{\mathrm{HCB}}\left(\mathbf{r} ; \mathbf{u}, \mathbf{u}^{\prime} ; \rho\right)=c_{\mathrm{HS}}\left(\frac{r}{\sigma\left(\hat{\mathbf{r}} ; \mathbf{u}, \mathbf{u}^{\prime}\right)} ; \eta\right]
$$

with $v_{0}$ the volume of the HCB and $\eta=v_{0} \rho$ the packing fraction. Insertion of Eqs. (2.6) and (2.7) into (2.5) yields

$$
\beta \phi_{\mathrm{ex}}[h]=-D 2^{D} \eta \int_{0}^{1} d \lambda(1-\lambda) \int_{0}^{\infty} d x x^{D-1} c_{\mathrm{HS}}(x ; \bar{\eta}[\lambda h]) \int d \mathbf{u} \int d \mathbf{u}^{\prime} h(\mathbf{u}) h\left(\mathbf{u}^{\prime}\right) \Sigma\left(\mathbf{u} \cdot \mathbf{u}^{\prime}\right)
$$


where

$$
\begin{aligned}
\Sigma\left(\mathbf{u} \cdot \mathbf{u}^{\prime}\right) & =\frac{1}{D 2^{D} v_{0}} \int d \widehat{\mathbf{r}}\left[\sigma\left(\widehat{\mathbf{r}} ; \mathbf{u}, \mathbf{u}^{\prime}\right)\right]^{D} \\
& =\frac{1}{2^{D} v_{0}} \int d \mathbf{r} \Theta\left(\sigma\left(\hat{\mathbf{r}} ; \mathbf{u}, \mathbf{u}^{\prime}\right)-r\right)
\end{aligned}
$$

is the ratio of the excluded volume of two HCB's of orientations $\mathbf{u}$ and $\mathbf{u}^{\prime}$ to that of two HS's of molecular volume $v_{0}[\boldsymbol{\Theta}(\boldsymbol{x})$ being the Heaviside step function]. At this level we can see that as a result of this approximation to the DCF [Eq. (2.7)] the excess free-energy factorizes into a radial and an angular contribution, leading to an Onsager-like theory [9]. Indeed, one recovers the original Onsager result by just replacing in (2.8) the DCF by its low-density limit $\left[c_{\mathrm{HS}}(x ; 0)=-\Theta(1-x)\right]$. Equation (2.8) can be further simplified by using some identities of the $D$-dimensional $\mathrm{HS}$ fluid [36]. If we let $Z_{\mathrm{HS}}(\eta)$ be the compressibility factor of $\mathrm{HS}\left[Z_{\mathrm{HS}}(\eta) \equiv \beta P_{\mathrm{HS}} / \rho, P_{\mathrm{HS}}\right.$ being the pressure of the HS fluid] and define $I_{\mathrm{HS}}(\eta)$ through the relation

$$
Z_{\mathrm{HS}}(\eta)=1-\frac{1}{\eta} \int_{0}^{\eta} d y y I_{\mathrm{HS}}(y)
$$

the compressibility equation of state $\left[\beta \partial P_{\mathrm{HS}} / \partial \rho\right.$ $\left.=1-\rho \int d \mathbf{r} c_{\mathrm{HS}}(r / \sigma ; \eta)\right]$ yields [36]

$$
I_{\mathrm{HS}}(\eta)=D 2^{D} \int_{0}^{\infty} d x x^{D-1} c_{\mathrm{HS}}(x ; \eta) .
$$

Substitution into Eq. (2.8) leads then to the following expression for $\phi_{\mathrm{ex}}[h]$, where the radial variables have now been integrated out:

$$
\begin{aligned}
\beta \phi_{\mathrm{ex}}[h]=-\eta \int_{0}^{1} d \lambda(1-\lambda) I_{\mathrm{HS}}(\bar{\eta}[\lambda h]) \\
\times \int d \mathbf{u} \int d \mathbf{u}^{\prime} h(\mathbf{u}) h\left(\mathbf{u}^{\prime}\right) \Sigma\left(\mathbf{u} \cdot \mathbf{u}^{\prime}\right) .
\end{aligned}
$$

To go ahead with the ELA we need a recipe to calculate the effective density $\bar{\rho}[h]$. In the earlier version of the ELA $[27,28]$ the effective density was determined from ad hoc geometric arguments. The problem of this method is that it is not at all obvious how to extend it to other phases. In the context of freezing this problem was solved by introducing a self-consistent ELA [35] (SCELA), which was later reformulated into a generalized ELA [7] (GELA). In this formalism the effective density $\bar{\rho}[\rho]$ is determined through a self-consistency relation between the excess free energy of the inhomogeneous (in our case anisotropic) phase $\phi_{\mathrm{ex}}[\rho]$ and that of the homogeneous phase evaluated at the effective density, $\phi_{\text {ex }}(\bar{\rho})$ (see Appendix). In the present case, due to the factorization approximation involved in (2.8), the selfconsistency relation can be imposed either on the total excess free energy or, as the angular part does not depend on the average density, on the radial part only. We will henceforth refer to these two different approaches as the total GELA ( $t$-GELA) and the radial GELA ( $r$-GELA), respectively, and denote the corresponding expressions with superscripts $t$ and $r$. For the $t$-GELA the selfconsistency relation reads $\phi_{\mathrm{ex}}[h]=\phi_{\mathrm{ex}}(\bar{\rho}[h])$. Thus the following effective packing fraction is obtained (see Appendix):

$$
\bar{\eta}^{t}[\lambda h]=\lambda \eta \frac{\int d \mathbf{u} \int d \mathbf{u}^{\prime} h(\mathbf{u}) h\left(\mathbf{u}^{\prime}\right) \Sigma\left(\mathbf{u} \cdot \mathbf{u}^{\prime}\right)}{\int d \mathbf{u} \int d \mathbf{u}^{\prime} \Sigma\left(\mathbf{u} \cdot \mathbf{u}^{\prime}\right)} .
$$

Note that $\bar{\rho}[\lambda \rho]=\lambda \bar{\rho}[\rho]$ is usually an approximate relation which takes us from the GELA back to the SCELA [7]. In our treatment of the $I-N$ transition Eq. (2.13) proves that the above relation turns out to be exact and so the GELA and the SCELA become equivalent. Inserting now (2.13) in (2.12) and using the self-consistency condition the resulting equation for the excess free energy per particle is

$$
\beta \phi_{\mathrm{ex}}^{t}[h]=Q_{\mathrm{HS}}\left(\bar{\eta}^{t}[h]\right)\left(\int d \mathbf{u} \int d \mathbf{u}^{\prime} \mathbf{\Sigma}\left(\mathbf{u} \cdot \mathbf{u}^{\prime}\right)\right)
$$

with $Q_{\mathrm{HS}}(\eta)$ defined by

$$
Q_{\mathrm{HS}}(x) \equiv \int_{0}^{x} d y \frac{Z_{\mathrm{HS}}(y)-1}{y} .
$$

Equation (2.14) is identical to the expression given by Holyst and Poniewierski [26] within the weighted-density formalism of Tarazona [37].

For the $r$-GELA we obtain $\bar{\eta}^{r}[\lambda h]=\lambda \eta$, and $\phi_{\mathrm{ex}}[h]$ reduces to

$$
\beta \phi_{\mathrm{ex}}^{r}[h]=Q_{\mathrm{HS}}(\eta)\left[\int d \mathbf{u} \int d \mathbf{u}^{\prime} h(\mathbf{u}) h\left(\mathbf{u}^{\prime}\right) \Sigma\left(\mathbf{u} \cdot \mathbf{u}^{\prime}\right)\right] .
$$

This equation is identical to the free-energy function proposed by Lee [30] on the basis of a scaling argument. However, the present formalism yields the same formula back in a natural way and makes clear how it can be obtained within the DFT. In the low-density limit $\left[Q_{\mathrm{HS}}(\eta)=\eta / 2\right]$ Eqs. (2.14) and (2.16) become identical to the original Onsager expression [9].

Once we have expressions for the free energy of a system of $D$-dimensional hard convex bodies we can obtain the other thermodynamic quantities such as the pressure $P$ or the chemical potential $\mu$, by starting from the exact DFT formulas [3]:

$$
\begin{aligned}
& \beta \frac{P[\rho]}{\rho}=1-\frac{1}{\rho V} \int d \mathbf{x} \int d \mathbf{x}^{\prime} \rho(\mathbf{x}) \rho\left(\mathbf{x}^{\prime}\right) \\
& \times \int_{0}^{1} d \lambda \lambda c\left(\mathbf{x}, \mathbf{x}^{\prime} ;[\lambda \rho]\right), \\
& \beta \mu[\rho]=\beta \frac{P[\rho]}{\rho}+\beta \phi[\rho] .
\end{aligned}
$$

Working out the expression (2.17) within the above approximation we end up with the following equations for the pressure:

$$
\begin{aligned}
\beta \frac{P^{t}[h]}{\rho}=1+ & \left\{Z_{\mathrm{HS}}\left(\bar{\eta}^{t}[h]\right)-1\right\}\left[\int d \mathbf{u} \int d \mathbf{u}^{\prime} \Sigma\left(\mathbf{u} \cdot \mathbf{u}^{\prime}\right)\right] \\
\beta \frac{P^{r}[h]}{\rho}=1+ & {\left[Z_{\mathrm{HS}}(\eta)-1\right] } \\
\times & \left(\int d \mathbf{u} \int d \mathbf{u}^{\prime} h(\mathbf{u}) h\left(\mathbf{u}^{\prime}\right) \Sigma\left(\mathbf{u} \cdot \mathbf{u}^{\prime}\right)\right] .
\end{aligned}
$$

The corresponding chemical potential can then be obtained from (2.18). 


\section{THE ONE-ORDER-PARAMETER APPROXIMATION}

To find the equilibrium density profile one takes the functional derivative of $\phi[h]$ while maintaining the normalization condition. In this way the Euler-Lagrange equation for the angular distribution function $h(\mathbf{u})$ is obtained. Since it is a highly nonlinear integral equation, approximate methods have to be used in order to get information out of it. One possible approach is to expand $h(\mathbf{u})$ - or better $\ln h(\mathbf{u})$ to guarantee the positiveness of $h(\mathbf{u})$ [38] - into an appropriate basis of orthogonal polynomials, truncate the expansion at some order, and then minimize the free energy with respect to the expansion coefficients taken as variational parameters. Although several of these parameters have to be retained in order to determine $h(\mathbf{u})$ as accurately as with a numerical method (for instance, that used in Ref. [40]), a single variational parameter is enough to get a good accuracy for the determination of the thermodynamic quantities [27]. This makes this method, henceforth referred to as the one-order-parameter (OOP) approximation, much simpler than the numerical one.

To implement the OOP approximation, we first have to determine which is the optimal basis of orthogonal polynomials to expand $\ln h(\mathbf{u})$ into. In $3 \mathrm{D}$ these polynomials are Legendre polynomials [27] and in 2D, Chebishev polynomials [28]. For a $D$-dimensional system such a basis is formed by

$$
M_{n}^{(D)}(x) \equiv\left(\begin{array}{c}
n+D-3 \\
n
\end{array}\right)^{-1} C_{n}^{[(D-2) / 2]}(x)
$$

where $C_{n}^{(\alpha)}(x)$ are the Gegenbauer ultraspherical polynomials [39], and the normalization is chosen such that $\left|M_{n}^{(D)}( \pm 1)\right|=1$. According to this choice the OOP distribution function $h(\mathbf{u}) \equiv h_{D}(\cos \theta)$ acquires the form

$h_{D}(\cos \theta)=\Omega_{D}(\gamma)^{-1} \exp \left[\gamma M_{2}^{(D)}(\cos \theta)\right]$,

$$
\Omega_{D}(\gamma) \equiv \int_{0}^{1} d x \frac{1}{N_{D}}\left(1-x^{2}\right)^{(D-3) / 2} \exp \left[\gamma M_{2}^{(D)}(x)\right]
$$

with

$$
N_{D} \equiv \int_{0}^{1} d x\left(1-x^{2}\right)^{(D-3) / 2}=\frac{\sqrt{\pi}}{2} \frac{\Gamma[(D-1) / 2]}{\Gamma(D / 2)} \text {. }
$$

As [39] $M_{2}^{(D)}(x)=\left(D x^{2}-1\right) /(D-1)$ we find that

$$
\begin{aligned}
\Omega_{D}(\gamma)=\frac{e^{\gamma}}{N_{D}}[ & {\left[-\frac{d}{d z}\right]^{|(D-2) / 2|} } \\
& \left.\times W_{(-)^{D}}(z)\right]_{z=\gamma D /(D-1)}
\end{aligned}
$$

where $\lfloor x\rfloor$ is the floor function, i.e., the largest integer less than or equal to $x$, and the $W_{ \pm}(z)$ are defined as

$$
\begin{aligned}
& W_{+}(z) \equiv \frac{\pi}{2} e^{-z / 2} I_{0}(z / 2), \\
& W_{-}(z) \equiv \frac{F(\sqrt{z})}{\sqrt{z}},
\end{aligned}
$$

$F(x)=e^{-x^{2}} \int_{0}^{x} d y e^{y^{2}}$ and $I_{0}(x)=\int_{0}^{\pi}(d \theta / \pi) e^{ \pm x \cos \theta}$ being the Dawson and the zeroth-order modified Bessel functions [39], respectively.

As in the 2D [28] and 3D [27] cases, the nematic order parameter, $q_{D}$ can be defined in terms of $M_{2}^{(D)}(\cos \theta)$

$$
q_{D}=\left\langle M_{2}^{(D)}(\cos \theta)\right\rangle=\frac{\partial}{\partial \gamma} \ln \Omega_{D}(\gamma)
$$

This equation makes clear the equivalence between the variational parameter $\gamma$ and the nematic order parameter $q_{D}$, justifying the name of the OOP approximation.

With the aid of all these definitions we are now ready to rewrite the free energy of the system in the OOP approximation. Thus, defining

$$
\begin{aligned}
H_{D}(\gamma)=\frac{2}{\pi^{3 / 2}} \frac{\Gamma(D / 2)}{\Gamma[(D-1) / 2]} \int_{0}^{1} d s \int_{0}^{1} d s^{\prime} \int_{-1}^{1} d x\left[\left(1-s^{2}\right)\left(1-s^{\prime 2}\right)\right]^{(D-3) / 2} \xi_{D}(x) \\
\\
\quad \times h_{D}(s) h_{D}\left(s^{\prime}\right) \Sigma\left[s s^{\prime}+\left(1-s^{2}\right)^{1 / 2}\left(1-s^{\prime 2}\right)^{1 / 2} \cos (\pi x)\right]
\end{aligned}
$$

with

$$
\zeta_{D}(x)= \begin{cases}(D-2)|\sin (\pi x)|^{D-3} & \text { if } D>2 \\ \delta(x) \text { if } D=2 & \end{cases}
$$

Eqs. (2.4), (2.14), and (2.16) become, respectively,

$$
\begin{aligned}
& \beta \phi_{\mathrm{id}}(\gamma)=\ln (\Lambda \rho)-1+\gamma q_{D}(\gamma)-\ln \Omega_{D}(\gamma), \\
& \beta \phi_{\mathrm{ex}}^{t}(\gamma)=Q_{\mathrm{HS}}\left[\bar{\eta}^{t}(\gamma)\right] H_{D}^{0}, \\
& \beta \phi_{\mathrm{ex}}^{r}(\gamma)=Q_{\mathrm{HS}}(\eta) H_{D}(\gamma),
\end{aligned}
$$

with $H_{D}^{0} \equiv H_{D}(0)$ and $\bar{\eta}^{t}(\gamma)=\eta H_{D}(\gamma) / H_{D}^{0}$. Minimization of $\phi(\gamma)=\phi_{\text {id }}(\gamma)+\phi_{\mathrm{ex}}(\gamma)$ as a function of $\gamma$ yields the curve $\gamma^{*}(\eta)$, which allows us to compute the pressure through one of the two equations

$$
\begin{aligned}
& \beta \frac{P^{t}}{\rho}=1+\left[Z_{\mathrm{HS}}\left(\bar{\eta}^{t}\left(\gamma^{*}(\eta)\right)\right)-1\right] H_{D}^{0}, \\
& \beta \frac{P^{r}}{\rho}=1+\left[Z_{\mathrm{HS}}(\eta)-1\right] H_{D}\left(\gamma^{*}(\eta)\right) .
\end{aligned}
$$

\section{THE EXCLUDED VOLUME OF TWO D-DIMENSIONAL HARD CONVEX BODIES}

The last input we need to complete our formalism is an expression for the excluded volume of two HCB's [Eq. (2.9)]. The most common HCB shapes appearing throughout the literature are prolate (rodlike) or oblate (disklike) HE's and prolate or oblate HSC's. The former are the immediate extensions to anisotropic shapes of the 
HS model, and hence they were one of the first systems for which the nematic phase was studied [14-18,20-24, 26-30]; the latter were introduced to study smectic phases $[12-14,24-26,30]$ because of their higher packing ability. Other HCB's have also been considered [41], but only very little and rather specific studies have been devoted to them. Also interesting are the limiting cases of extremely high prolateness (needles $[11,28]$ ) or oblateness (platelets $[10,27]$ ), because for these the nematic phase is not disturbed by the presence of a solid phase (needles and platelets have no molecular volume). Finally, HE's with three distinct axes have also been proposed to simulate biaxial phases [16].

Since the analytical expression for the excluded volume of two $D$-dimensional HE's with given orientations $u$ and $\mathbf{u}^{\prime}$ has not yet been given for any $D$, an approximate analytical expression for this excluded volume can be obtained from the so-called Gaussian-overlap approximation [42] (GOA). This expression is

$$
\Sigma^{\mathrm{GOA}}\left(\mathbf{u} \cdot \mathbf{u}^{\prime}\right)=\left(\frac{1-\chi^{2}\left(\mathbf{u} \cdot \mathbf{u}^{\prime}\right)^{2}}{1-\chi^{2}}\right)^{1 / 2}
$$

where $\chi$ is related to the aspect ratio $\kappa=\sigma_{\|} / \sigma_{\perp}$ by $\chi=\left(\kappa^{2}-1\right) /\left(\kappa^{2}+1\right)$. The formula (4.1) is valid provided $D \geq 2$ [43]

Notice that Eq. (4.1) has oblate-prolate symmetry, since it depends on $\chi^{2}(\chi<0$ for oblate ellipsoids and $\chi>0$ for prolate ellipsoids), so that Eqs. (2.14) and (2.16) will lead to the same thermodynamics for oblate and prolate HE's. As this is in general only approximately true [15] (except for $2 \mathrm{D}$, where the symmetry is obviously exact) this feature appears as a defect of the theory. However, the GOA itself is not responsible for this effect because the exact second virial coefficient also has this oblate-prolate symmetry. The origin of this failure can be found again in the approximation [Eq. (2.7)] of the DCF, which leads to an Onsager-like theory that only takes into account the second-order virial contribution with respect to the angular variables.

The GOA has been widely used throughout the literature $[22,23,27-30]$. Its main difficulty is that it overestimates the real excluded volume of two HE's [21], yielding a second virial coefficient different from the exact one $[18,41]$. The second virial coefficient in the GOA is [43]

$$
\frac{B_{2}^{\mathrm{GOA}}}{v_{0}}=2^{D-1} \frac{F\left(-\frac{1}{2}, \frac{1}{2} ; D / 2 ; \chi^{2}\right)}{\left(1-\chi^{2}\right)^{1 / 2}}
$$

where $F(\alpha, \beta ; \gamma ; z)$ is the hypergeometric function [39]. A slight improvement can be obtained [18] if we rescale (4.1) by the factor $B_{2}^{\text {exact }} / B_{2}^{\mathrm{GOA}}$, whenever the exact second virial coefficient $B_{2}^{\text {exact }}$ is available. In the following, all calculations involving the excluded volume of two HE's will be performed with the help of the GOA (rescaled when possible).

For HSC's the problem is much simpler [44]. The parameters defining a HSC are the length of the cylinder, $L$, and the diameter of the spherical caps, $W$. The aspect ratio (the length-to-width ratio) can then be defined as $\kappa=1+L / W$. In $2 \mathrm{D}$, the excluded volume (area) is given by the area of the figure shown in Fig. 1. It is composed by the sum of the area of four rectangles of side lengths $L$ and $W$, four circle sectors which form a whole circle of radius $W$, and a parallelogram of length $L$ and angle $\theta$, where $\mathbf{u} \cdot \mathbf{u}^{\prime}=\cos \theta, \mathbf{u}$ and $\mathbf{u}^{\prime}$ being the orientations of the two HSC's. Thus

$$
V_{\mathrm{excl}}^{(2-\mathrm{HSC})}(\theta)=4 L W+\pi W^{2}+L^{2}|\sin \theta| .
$$

From Fig. 1 it can be inferred that in 3D the rectangles are replaced by half cylinders of height $L$ and radius $W$, the circle sectors become spherical caps with the same radius, and the parallelogram gains a thickness $2 W$ becoming a parallelepiped, so in this case

$V_{\mathrm{excl}}^{(3-\mathrm{HSC})}(\theta)=2 L \pi W^{2}+\frac{4}{3} \pi W^{3}+2 W L^{2}|\sin \theta|$.

The step from 2D to 3D illustrates how to go to a higher dimension, so that for a $D$-dimensional system we can induce [45]:

$$
\begin{aligned}
V_{\mathrm{excl}}^{(D-\mathrm{HSC})}(\theta)= & 2 L V_{D-1} W^{D-1}+V_{D} W^{D} \\
& +V_{D-2} W^{D-2} L^{2}|\sin \theta|
\end{aligned}
$$

where $V_{n}$ denotes the volume of an $n$-dimensional sphere of unit radius, i.e., $V_{n}=\pi^{n / 2} / \Gamma(1+n / 2)$. Now, if we take into account that the volume of one of these HSC's is $v_{0}=V_{D}(W / 2)^{D}+L V_{D-1}(W / 2)^{D-1}$, the reduced excluded volume (2.9) takes the form

$$
\begin{aligned}
\Sigma^{\mathrm{HSC}}\left(\mathbf{u} \cdot \mathbf{u}^{\prime}\right)=1+ & \frac{D}{2 \pi} \frac{(\kappa-1)^{2}}{\left[1+(\kappa-1) / N_{D+2}\right]} \\
& \times\left[1-\left(\mathbf{u} \cdot \mathbf{u}^{\prime}\right)^{2}\right]^{1 / 2}
\end{aligned}
$$

with $N_{D}$ given by (3.4), thus leading to the following second virial coefficient:
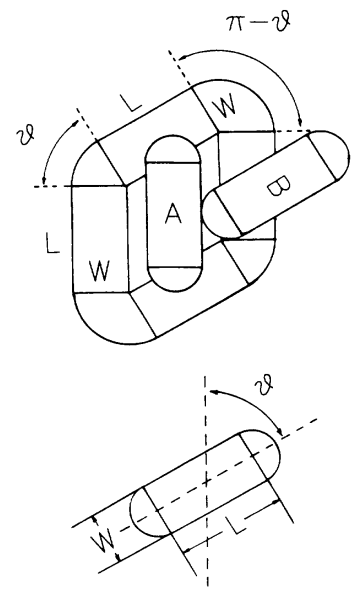

FIG. 1. Excluded area of two 2D hard spherocylinders (circle-rectangles) with relative orientation $\theta$, or, equivalently, projection on the plane containing the symmetry axes of the excluded volume of two $3 \mathrm{D}$ hard spherocylinders with the same relative orientation. The figure is described by the center of mass of particle $B$ as it moves around particle $A$ while keeping contact at one point at least. $L$ is the length of the cylindrical bodies and $W$ is their width, as well as the diameter of the spherical caps. 


$$
\frac{B_{2}^{\mathrm{HSC}}}{v_{0}}=2^{D-1}\left(1+\frac{D}{4} \frac{(\kappa-1)^{2}}{\left[(D-1) N_{D}^{2}+D N_{D}(\kappa-1)\right]}\right]
$$

Notice that $\lim _{\kappa \rightarrow 0} \kappa \Sigma^{\mathrm{GOA}}\left(\mathbf{u} \cdot \mathbf{u}^{\prime}\right) \propto \Sigma^{\mathrm{HSC}}\left(\mathbf{u} \cdot \mathbf{u}^{\prime}\right)-1$ so that the behavior of a system of HSC's is essentially contained
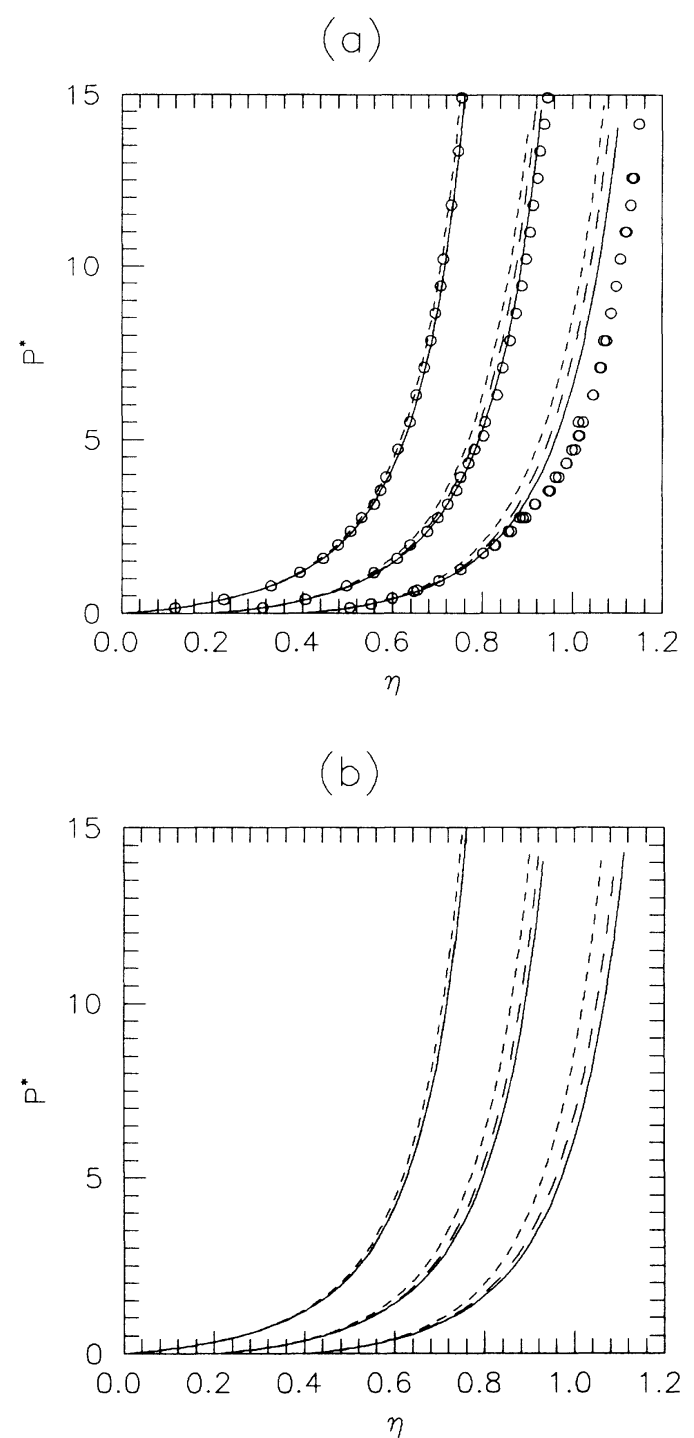

FIG. 2. Reduced pressure $P^{*} \equiv \beta P v_{0}$ of (a) 2D HE's (hard ellipses) and (b) 2D HSC's, as a function of the packing fraction $\eta \equiv v_{0} \rho$, for aspect ratios $\kappa=2,4$, and 6 (from left to right). Solid lines represent Eq. (5.1) with $Z_{6}$ [36] as reference HS EOS; long-dashed lines show the SPT results [47]; short-dashed lines show Song and Mason's EOS [46] with the data of Tarjus et al. [49] for the virial coefficients, and circles in (a) are MC simulations for hard ellipses [18] (there are no available simulation results for circle-rectangles). The curves for $\kappa=4$ and 6 are shifted 0.2 and 0.4 units to the right, respectively. For $\kappa=2$ solid and long-dashed lines coincide. In (a) there is a KosterlitzThouless transition to a nematic phase [18] for hard ellipses with $\kappa=6$ at $\eta=0.59$; besides, there is an apparent first-order transition at $\eta=0.51$ due to the finite size of the system, so data above these values have to be considered with care. in the limit of very large eccentricity of a system of HE's, as we will see later on, in Sec. VIII.

\section{THE ISOTROPIC PHASE}

The equation of state (EOS) for the isotropic phase is obtained by setting $\gamma^{*}=0$ in (3.14) and (3.15). Both
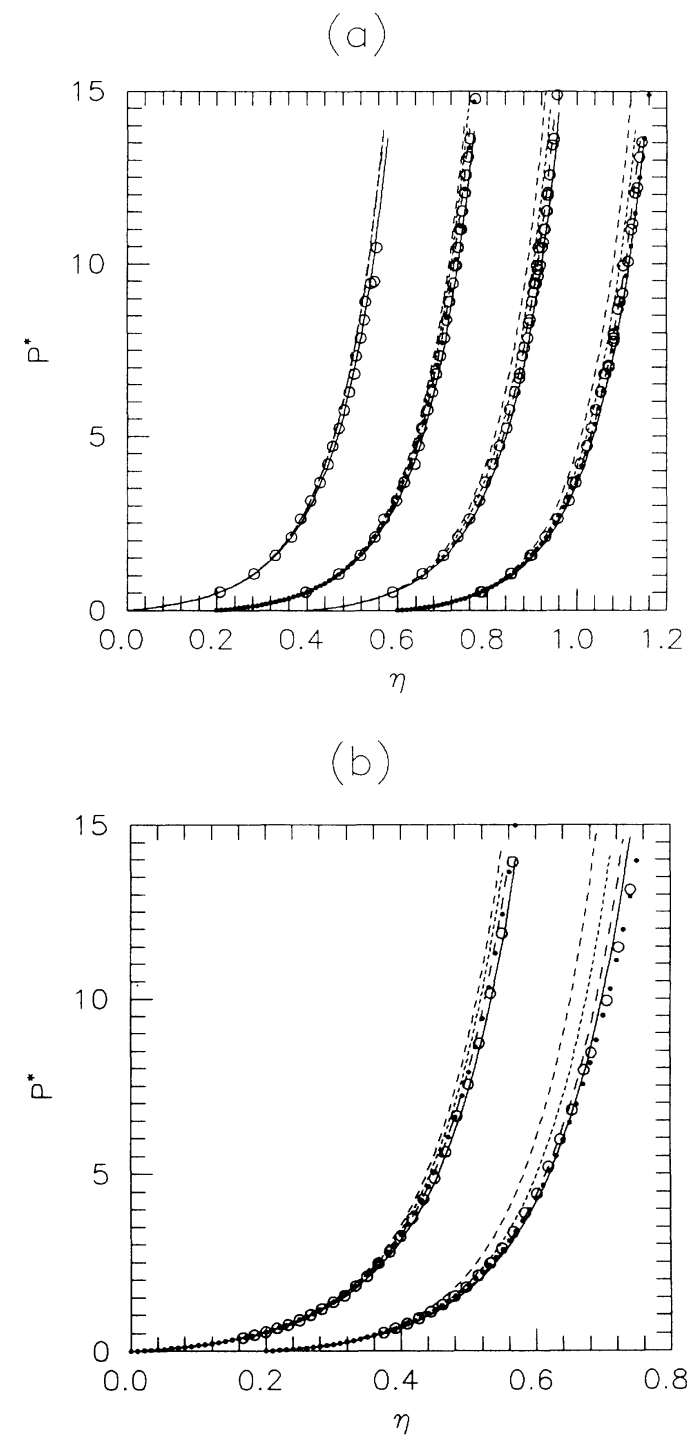

FIG. 3. Reduced pressure $P^{*} \equiv \beta P v_{0}$ of (a) 3D HE's as a function of the packing fraction $\eta \equiv v_{0} \rho$, for aspect ratios $\kappa=1.25,2,2.75$, and 3 (from left to right), and (b) 3D HSC's, for length-to-width ratios $\kappa=2$ and 4. Curves are obtained from Eq. (5.1), using as HS EOS the CS (solid lines) and the PY-c (long dashed lines), from the SPT of Boublík [47] (mediumdashed lines), from the scaled-field particle (SFP) theory of Rosenfeld [48] (short-dashed lines), and from Song and Mason's proposal [46] (dots; only when numerical values for the virial coefficients are available). In (a) circles represent MC simulations [15]; the curves for $\kappa=2,2.75$, and 3 are shifted $0.2,0.4$, and 0.6 units to the right, respectively. In (b) circles represent MD simulations of Veerman and Frenkel [13]; the curves for $\kappa=4$ are shifted 0.2 units to the right. 
equations lead to the same result, that is,

$$
\beta \frac{P_{I}}{\rho}=1+H_{D}^{0}\left(Z_{\mathrm{HS}}(\eta)-1\right) .
$$

This expression is the obvious generalization to a system of $D$-dimensional HCB's of those previously obtained in 2D [28] and 3D [27], and it is again a direct consequence of the approximation used for the DCF [Eq. (2.7)]. Notice that we have not made use of the explicit expression of the DCF, so we are totally free to use more accurate EOS's for the HS reference system than the simple Percus-Yevick (PY) [6].

It is interesting to compare the results of Eq. (5.1) with other EOS's which have been proposed for the isotropic HCB fluid, namely, the Song and Mason proposal [46], based upon the knowledge of the three first virial coefficients; the scaled-particle theory (SPT) $[47,48]$, and, of course, the available simulation results. This comparison is done in Figs. 2 and 3 for $D=2$ and 3, respectively. Also interesting is to compare the virial coefficients $B_{n}^{\mathrm{HCB}}$ predicted by these theories with the numerical estimations for the exact ones. From Eq. (5.1) it is straightforward to show that

$$
b_{n}^{\mathrm{HCB}}=\left(H_{D}^{0}\right)^{-(n-1)} b_{n}^{\mathrm{HS}}
$$

where $b_{n} \equiv B_{n+1} /\left(B_{2}\right)^{n}$ are the reduced virial coefficients. From this expression it follows:

$$
\lim _{\kappa \rightarrow \infty} b_{n}^{\mathrm{HCB}}=0, \quad n \geq 2 .
$$

This property was proven to hold for $D=3$ (and it can also be proved for $D \geq 3$ ) by Onsager [9], and indeed all theories verify it except the SPT of Boublik [47], for which $b_{2}^{\mathrm{SPT}} \rightarrow \frac{1}{3}$ as $\kappa \rightarrow \infty$. However, although for $D=2$ all the theories satisfy (5.3), it has been proven to be false

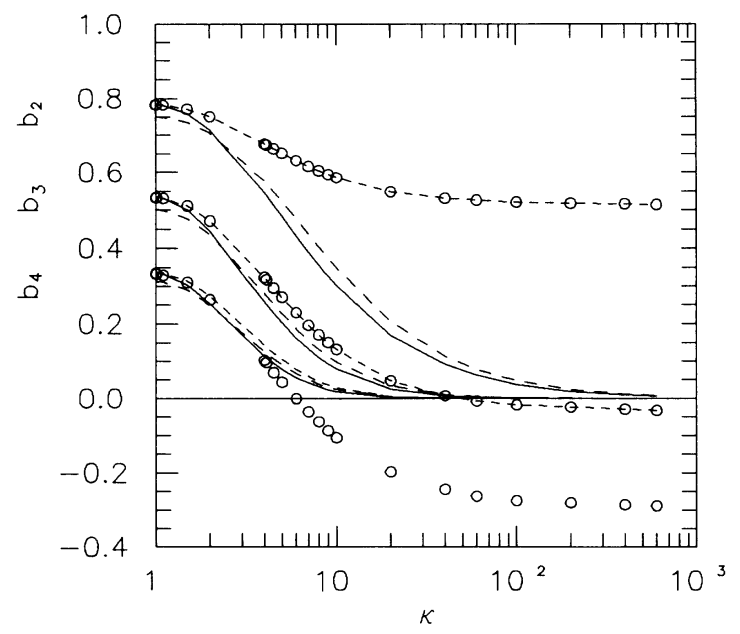

FIG. 4. Reduced virial coefficients, $b_{n} \equiv B_{n+1} /\left(B_{2}\right)^{n}(n=2,3$ and 4 , from top to bottom), vs the aspect ratio $\kappa$, for a system of 2D HE's. Circles represent the numerical results of Refs. [49] and [50]; solid lines are the predicted virial coefficients after Eq. (5.2); long-dashed lines are the SPT results of Ref. [47], and short-dashed lines are Song and Mason's results [46] (recall that $b_{2}$ and $b_{3}$ are inputs in their theory).
$[11,49]$. In Figs. 4 and 5 the results for $b_{2}, b_{3}$, and $b_{4}$ predicted for all these theoretical approaches are compared to the numerical estimations for 2D $[49,50]$ and $3 D$ [51] HE's, respectively. This fact makes clear a profound failure of the theory for $D=2$ suggesting that the simple approximation for the DCF [Eq. (2.7)], which is responsible for the form (5.2) of the approximate virial coefficients, is not valid for 2D HCB systems [29].
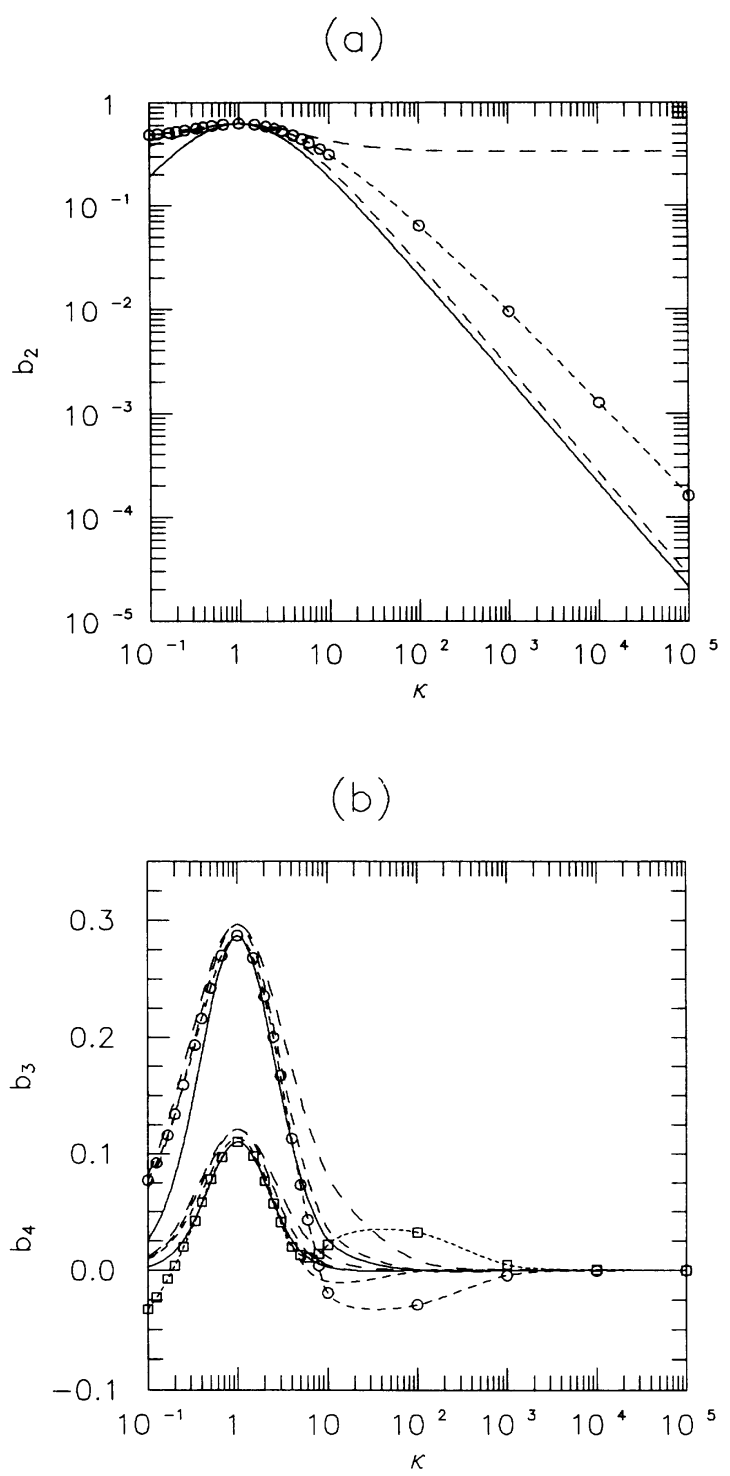

FIG. 5. Reduced virial coefficients, $b_{n} \equiv B_{n+1} /\left(B_{2}\right)^{n}$, vs the aspect ratio $\kappa$, for a system of 3D HE's. Circles and squares represent the numerical results of Ref. [51]; solid lines are the predicted virial coefficients after Eq. (5.2); long-dashed lines are the SPT results of Ref. [47]; medium-dashed lines are the SFP results of Ref. [48], and short-dashed lines are Song and Mason's results [46] (recall that $b_{2}$ and $b_{3}$ are inputs in their theory). (a) is a log-log plot of $b_{2}$. For large $\kappa$ the slopes of the solid and medium-dashed lines are 1 while that of the shortdashed line is nearly 0.9. (b) is a plot of $b_{3}$ and $b_{4}$. The very short-dashed line joining the squares has been drawn as a guide to the eye. 


\section{THE NEMATIC PHASE OF D-DIMENSIONAL HARD ELLIPSOIDS}

Minimizing $\phi(\gamma)=\phi_{\mathrm{id}}(\gamma)+\phi_{\mathrm{ex}}(\gamma)$ with respect to $\gamma$, with $\phi_{\text {id }}(\gamma)$ given by (3.11) and $\phi_{\mathrm{ex}}(\gamma)$ given by either (3.12) or (3.13), we find a unique solution, $\gamma=0$, for packing fractions up to a certain value, $\eta_{0}$. This means that the isotropic phase is the only stable phase in this region. However, above $\eta_{0}$, a second minimum, $\gamma^{*}(\eta) \neq 0$, coexists with the former. It corresponds to the appearance of a nematic phase. $\eta_{0}$ and $\gamma^{*}(\eta)$ depend on which version of the GELA (total or radial) we choose. For $D=2$, $\gamma^{*}(\eta)$ goes to zero as we approach $\eta_{0}$ from above, so that at $\eta_{0}$ both solutions meet. Further, the isotropic phase becomes unstable above $\eta_{0}$ and so there is no coexistence of the two phases for $\eta \neq \eta_{0}$. The transition is, thus, second order, in agreement with the previous findings [28] and for the same reason, i.e., the Landau expansion is an even function of the order parameter, so no thirdorder term appears, and the fourth-order term is always positive, prohibiting the existence of a tricritical point. As pointed out in Ref. [18], this conclusion seems to be in strong disagreement with the simulations performed on 2D HE's, where the $I-N$ transition is of the KosterlitzThouless type for high eccentricities, and first order for low eccentricities. For $D>2, \gamma^{*}(\eta)$ is discontinuous at $\eta_{0}$, and above this value up to $\eta_{2}>\eta_{0}$, where the $\gamma=0$ solution becomes unstable, both solutions are local minima of the free energy. The magnitude of $\phi(\gamma)$ for each minimum is what determines which of them is the absolute minimum, and hence distinguishes the truly stable phase from the metastable one. Eventually both phases reach the same value of the free energy at a certain packing fraction, $\eta_{1}\left(\eta_{0}<\eta_{1}<\eta_{2}\right)$. All this phenomenology corresponds to a first-order transition. For $D=3$ this conclusion agrees with the simulations on HE's [15]; however, the simulations on HSC's [13] are not conclusive enough to distinguish between a continuous transition or a very weak first-order transition.

The above conclusions arise by working out the OOP approximation (see Sec. III) numerically. However, some results can also be proven analytically. The order of the transition can be obtained from a Landau expansion of the free energy in terms of the order parameter $q_{D}$ $[27,28]$, and the stability limit of the isotropic phase, $\eta_{2}$, is found from the change of sign of the second derivative of the free energy at $\gamma=0\left[\phi^{\prime \prime}(0)<0\right.$ implies stable isotropic and $\phi^{\prime \prime}(0)>0$ unstable isotropic phases]. After some algebra and making use of the summation theorem of the Gegenbauer polynomials [39] the stability conditions for the $t$-GELA and the $r$-GELA read, respectively,

$$
\begin{aligned}
& 2\left[Z_{\mathrm{HS}}\left(\eta_{2}^{t}\right)-1\right] \int d \mathbf{u} M_{2}^{(D)}(\mathbf{u}) \Sigma(\mathbf{u})+1=0, \\
& 2 Q_{\mathrm{HS}}\left(\eta_{2}^{r}\right) \int d \mathbf{u} M_{2}^{(D)}(\mathbf{u}) \Sigma(\mathbf{u})+1=0 .
\end{aligned}
$$

As we have just seen, the isotropic and the nematic phases coexist within an interval of densities around the transition point. The phase equilibrium conditions, i.e., $P_{i}=P_{n}$ and $\mu_{i}=\mu_{n}$, with $P_{i}\left(P_{n}\right)$ the pressure and $\mu_{i}\left(\mu_{n}\right)$ the chemical potential of the isotropic (nematic) phase can then be solved in this region. The solution to this system of equations yields thus $\eta_{i}$ and $\eta_{n}$, the packing fractions of the isotropic and the nematic phases at coexistence.

In Fig. 6 we plot the EOS of 2D HE's obtained from the $t$-GELA and the $r$-GELA, together with the Monte Carlo (MC) results of Ref. [18], for aspect ratio $\kappa=2,4$, and 6 . We can see that, although the prediction of the order (always second order) and the location of the transition (always too low) are wrong (see also Fig. 7), the numerical values of the EOS obtained from the $r$-GELA (with $Z_{6}$ of Baus and Colot [36] as HS EOS) are not very far from the simulations. Besides, for $\kappa=2$, where according to the simulations the nematic phase is preempted by a more ordered phase (presumably a solid), the transition point obtained from this approach is placed above the density at which this new phase appears. This suggests that the $r$-GELA is reliable to describe the thermodynamics of the system for not too elongated 2D HE's. However, the $t$-GELA predicts a nematic branch which deviates excessively from the MC data. Even the

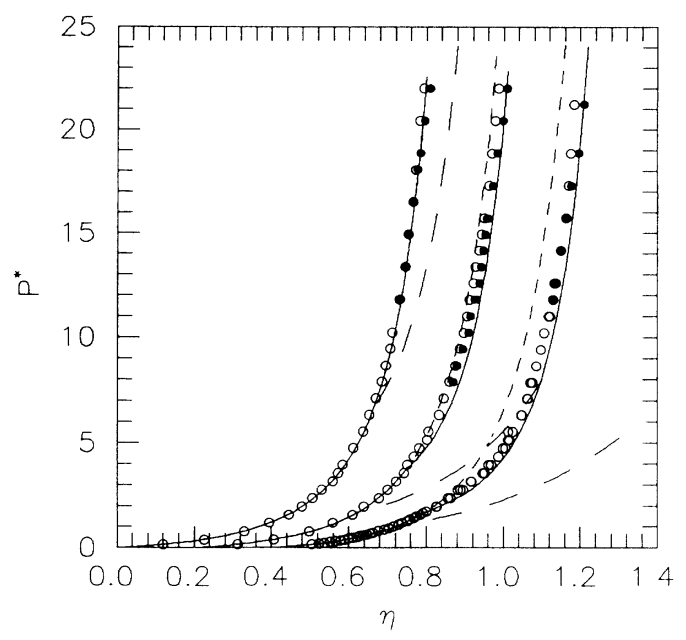

FIG. 6. Reduced pressure $P^{*} \equiv \beta P v_{0}$ of 2D HE's (hard ellipses) as a function of the packing fraction $\eta \equiv v_{0} \rho$, for aspect ratios $\kappa=2,4$, and 6 (from left to right, each shifted 0.2 units to the right). Solid lines show the pressure at the stable phase (either the isotropic or the nematic) as obtained from the $r$-GELA. Short-dashed lines show the pressure of the unstable isotropic phase. Long-dashed lines represent the pressure of the nematic phase as obtained from the $t$-GELA. Empty circles are the MC results given by Ref. [18], as computed starting from a lowdensity configuration. For $\kappa=2$ and 4 they correspond to the isotropic phase. For $\kappa=6$ they belong to the nematic phase above $\eta=0.59$, where a Kosterlitz-Thouless transition takes place. Filled circles are the same MC results but this time computed starting from a high-density configuration. Above $\eta=0.78,0.79$, and 0.76 , for $\kappa=2,4$, and 6 , respectively, another phase (presumably a solid) exists. Below those densities the filled and empty circles coincide for $\kappa=2$ and 6 , but for $\kappa=4$ they belong to a nematic phase which goes over into an isotropic phase at $\eta=0.74$. Notice the strong disagreement between the nematic pressure obtained from the $t$-GELA and the simulations. 


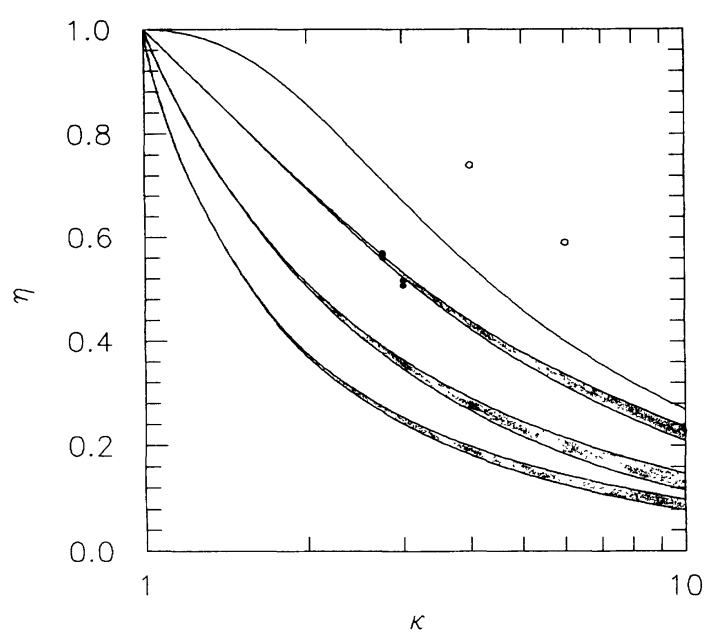

FIG. 7. Transition lines for the $I-N$ transition of $D$ dimensional HE, for $D=2,3,4$, and 5 (from top to bottom) as obtained from the $r$-GELA. For $D=2$ the $I-N$ transition is second order, whereas for $D>2$ it is first order. The shaded areas of the first-order transitions indicate the coexistence region. Empty circles correspond to the 2D transition as obtained from simulations [18]. In this case, for $\kappa=2$ and below, the nematic phase is preempted by another phase (presumably a solid). Filled circles correspond to the 3D transition as obtained from sirrulations [15]. Notice the good agreement with the present calculations. The nematic phase is always placed above the lines while the isotropic is below the lines.

location of the transition is lower than that obtained from the $r$-GELA. In particular, for $\kappa=2$ the transition is predicted where the simulations clearly show an isotropic fluid. In the case of 3D HE's the r-GELA yields very accurate results for the nematic branch, when compared with the simulations of Ref. [15] (see Fig. 8), and provides values for the location of the transition which are in excellent agreement with the simulations (see Fig. 7 and Table I). On the contrary, the $t$-GELA exhibits the same failure as in 2D. For this reason we will henceforth only consider the $r$-GELA, although eventually we will come back to the $t$-GELA but mentioning it explicitly. In Fig. 8 we also show the dependence of the results for the pressure on the HS EOS used in (3.15). Three different choices [Carnahan Starling (CS), Baus-Colot's $Z_{4}$ [36], and SPT-identical to the compressibility PY (PY-c)] have been made. Results do not strongly depend on which of them is used; however, SPT values seem to fit the MC data slightly better. Table I shows a comparison between the coexistence results obtained from the $r$ GELA with the three mentioned HS EOS's, from the $t$ GELA, from the MC simulations [15], and from other theoretical approaches found in the literature. Again there is not much influence of the choice of the HS EOS. Lee's theory [30] is based upon the functional (2.16) $(r$ GELA) but solving numerically the Euler equation for $h(\mathbf{u})$. The difference between his results and those of our $r$-GELA are due to this fact and to our rescaling of the excluded volume (see Sec. IV). Furthermore, Holyst and Poniewierski's theory [26] is based upon functional (2.14)

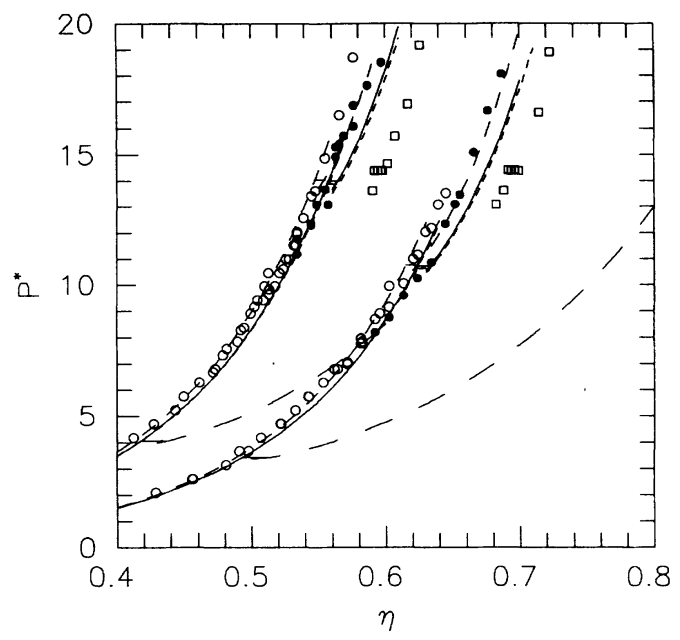

FIG. 8. Reduced pressure $P^{*} \equiv \beta P v_{0}$ of $3 \mathrm{D}$ HE as a function of the packing fraction $\eta \equiv v_{0} \rho$, for aspect ratios $\kappa=2.75$ and 3 (from left to right, each shifted 0.2 units to the right). Solid lines show the pressure at the isotropic and the nematic phases as obtained from the r-GELA using the CS EOS for the HS reference system. Medium- and short-dashed lines are the same, but using the SPT and the $Z_{4}$ of Ref. [36] as EOS for the HS reference system. Long-dashed lines represent the pressure of the nematic phase as obtained from the $t$-GELA with the CS HS EOS. Empty circles, filled circles, and squares are the MC results of Ref. [15] for the isotropic, nematic, and solid phases, respectively. Tie-lines show the coexistence obtained from the corresponding approaches. As in 2D (Fig. 6) the nematic branches obtained from the $t$-GELA are in strong disagreement with the simulations.

$(t$-GELA), and their results and those obtained from our $t$-GELA are much poorer than the former. Also worth noticing is the superiority of the $r$-GELA for reproducing the thermodynamics of the transition, namely, the pressure and chemical potential, at coexistence.

Results for the stability limits of the nematic and the isotropic phases ( $\eta_{0}$ and $\eta_{2}$, respectively) for a system of $D$-dimensional HE's are given in Table II. Also listed in it are the values of the order parameter $q_{D}$, as well as the difference between the free energy of the two phases, at those values of $\eta$. It can be seen that the order parameter is a slowly varying function of the aspect ratio $\kappa$. This feature has been interpreted as a Lindemann rule for the $I-N$ transition [27]. The difference of free energy is also a slowly varying function of $\kappa$. It is very small for $D=3$, but increases very rapidly with $D$. The results obtained from the coexistence equations are listed in Table III, and a plot of the transition lines (with its corresponding widths for the first-order transitions) is shown in Fig. 7. These results show that the $I-N$ transition starts being rather narrow at low eccentricities $\left(\Delta \eta / \eta_{n}\right.$, with $\Delta \eta=\eta_{n}-\eta_{i}$, is less than $10 \%$ up to $\kappa \sim 4$, for all $D$ ) but it widens as $\kappa$ increases, as could be expected. The jump in the order parameter $q_{D}$ is always quite strong, against the idea of the $I-N$ being a weakly first-order transition and invalidating the possibility of a converging Landau expansion. 
TABLE I. Data for the coexistence of the isotropic and the nematic phases of a system of 3D HE's with aspect ratio $\kappa$, as obtained by Monte Carlo (MC) simulations [15], by the r-GELA [with Carnahan Starling (CS) [6], Baus-Colot's $Z_{4}$ [36] and SPT, or equivalently PY-c [6], HS EOS's] and the $t$-GELA (with CS HS EOS), by numerical solution of (2.16) as reported by Lee [30] and by other previous theories, namely, those of Marko [23], Baus et al. [27], Holyst and Poniewierski [26] [whose calculations are based upon the functional (2.14)], Mulder and Frenkel [20], Perera, Patey, and Weis [24], and Singh and Singh [22]. $\eta_{i}$ and $\eta_{n}$ denote the packing fraction of the isotropic and nematic phases, respectively; $\Delta \eta=\eta_{n}-\eta_{i} ; P^{*}=\beta P v_{0}$ and $\mu_{\mathrm{ex}}^{*}=\beta \mu_{\mathrm{ex}}$ are the reduced pressure and the excess chemical potential at the coexistence point, and $q$ is the order parameter of the nematic phase at $\eta_{n}$.

\begin{tabular}{|c|c|c|c|c|c|c|}
\hline & $\kappa$ & $\eta_{i}$ & $\Delta \eta$ & $P^{*}$ & $\mu_{\mathrm{ex}}^{*}$ & $q$ \\
\hline \multirow[t]{6}{*}{$\mathrm{MC}$} & 3 & 0.507 & 0.010 & 9.79 & 25.15 & \\
\hline & $1 / 3$ & 0.498 & 0.010 & 9.15 & 24.03 & \\
\hline & 2.75 & 0.561 & 0.009 & 15.70 & 35.68 & \\
\hline & $1 / 2.75$ & 0.545 & 0.014 & 13.45 & 41.69 & \\
\hline & 2 & $(0.620)$ & & $(24.59)$ & $(49.03)$ & \\
\hline & $1 / 2$ & $(0.615)$ & & (23.96) & $(48.23)$ & \\
\hline$r$-GELA & 3 & 0.523 & 0.009 & 10.72 & 28.1 & 0.536 \\
\hline \multirow[t]{2}{*}{ with CS } & 2.75 & 0.557 & 0.008 & 14.00 & 33.7 & 0.522 \\
\hline & 2 & 0.693 & 0.003 & 50.10 & 88.3 & 0.477 \\
\hline$r$-GELA & 3 & 0.524 & 0.009 & 10.64 & 28.0 & 0.538 \\
\hline with $Z_{4}$ & 2.75 & 0.558 & 0.008 & 13.88 & 33.5 & 0.523 \\
\hline$r$-GELA & 3 & 0.515 & 0.009 & 10.77 & 28.5 & 0.538 \\
\hline with SPT (PY-c) & 2.75 & 0.547 & 0.007 & 14.05 & 34.3 & 0.523 \\
\hline$t$-GELA & 3 & 0.394 & 0.020 & 3.43 & 12.8 & 0.488 \\
\hline \multirow[t]{2}{*}{ with CS } & 2.75 & 0.417 & 0.019 & 4.06 & 14.1 & 0.480 \\
\hline & 2 & 0.523 & 0.011 & 8.97 & 25.3 & 0.453 \\
\hline \multirow[t]{2}{*}{ Ref. [30] } & 3 & 0.508 & 0.009 & 10.00 & 25.63 & 0.533 \\
\hline & 2.75 & 0.544 & 0.008 & 13.19 & 31.22 & 0.517 \\
\hline \multirow[t]{2}{*}{ Ref. [23] } & 3 & 0.493 & 0.001 & & & 0.017 \\
\hline & 2.75 & 0.517 & & & & 0.010 \\
\hline \multirow[t]{3}{*}{ Ref. [27] } & 3 & 0.472 & 0.012 & 7.76 & 22.3 & 0.561 \\
\hline & 2.75 & 0.501 & 0.011 & 9.62 & 25.7 & 0.548 \\
\hline & 2 & 0.612 & 0.006 & 24.3 & 50.2 & 0.50 \\
\hline \multirow[t]{2}{*}{ Ref. [26] } & 3 & 0.454 & 0.02 & 4.68 & & 0.485 \\
\hline & 2.75 & 0.475 & 0.018 & 5.48 & & 0.477 \\
\hline \multirow[t]{6}{*}{ Ref. [20] } & 3 & $0: 420$ & 0.018 & 5.31 & & 0.568 \\
\hline & $1 / 3$ & 0.413 & 0.017 & 5.27 & & 0.546 \\
\hline & 2.75 & 0.449 & 0.015 & 6.55 & & 0.553 \\
\hline & $1 / 2.75$ & 0.443 & 0.015 & 6.52 & & 0.550 \\
\hline & 2 & 0.576 & 0.007 & 17.84 & & 0.498 \\
\hline & $1 / 2$ & 0.573 & 0.007 & 17.86 & & 0.497 \\
\hline \multirow[t]{2}{*}{ Ref. [24] } & 3 & 0.418 & 0.018 & & & 0.657 \\
\hline & $1 / 3$ & 0.405 & 0.015 & & & 0.638 \\
\hline \multirow[t]{3}{*}{ Ref. [22] } & 3 & 0.309 & 0.021 & & & 0.547 \\
\hline & 2.75 & 0.329 & 0.018 & & & 0.532 \\
\hline & 2 & 0.415 & 0.008 & & & 0.480 \\
\hline
\end{tabular}


TABLE II. Metastability limits of the isotropic and the nematic phases of a system of $\boldsymbol{D}$-dimensional HE's as obtained from (3.13). For $\eta<\eta_{0}\left(\eta>\eta_{2}\right)$ the nematic (isotropic) phase is unstable. $q_{D}^{(0)}\left(q_{D}^{(2)}\right)$ and $\Delta f^{(0)}\left(\Delta f^{(2)}\right)$ are the order parameter of the nematic phase and the difference between the free energy of the nematic phase and that of the isotropic phase, computed at $\eta_{0}\left(\eta_{2}\right)$. Notice that these two latter quantities are not very sensitive to changes in the aspect ratio $\kappa$, but they increase very quickly with $D$. We have used CS as the reference HS EOS.

\begin{tabular}{|c|c|c|c|c|c|c|c|}
\hline$D$ & $\kappa$ & $\eta_{0}$ & $q_{D}^{(0)}$ & $\beta \Delta f^{(0)} / \rho$ & $\eta_{2}$ & $q_{D}^{(2)}$ & $\beta \Delta f^{(2)} / \rho$ \\
\hline \multirow[t]{6}{*}{3} & 2 & 0.6926 & 0.344 & 0.00458 & 0.7096 & 0.632 & -0.0744 \\
\hline & 3 & 0.5248 & 0.365 & 0.00507 & 0.5482 & 0.654 & -0.0835 \\
\hline & 4 & 0.4269 & 0.372 & 0.00559 & 0.4521 & 0.672 & -0.0917 \\
\hline & 5 & 0.3629 & 0.382 & 0.00594 & 0.3881 & 0.685 & -0.0980 \\
\hline & 6 & 0.3173 & 0.378 & 0.00631 & 0.3420 & 0.696 & -0.1036 \\
\hline & 10 & 0.2157 & 0.408 & 0.00685 & 0.2366 & 0.720 & -0.1155 \\
\hline \multirow[t]{6}{*}{4} & 2 & 0.4826 & 0.445 & 0.0298 & 0.5252 & 0.778 & -0.503 \\
\hline & 3 & 0.3302 & 0.472 & 0.0331 & 0.3766 & 0.802 & -0.566 \\
\hline & 4 & 0.2541 & 0.486 & 0.0361 & 0.2990 & 0.820 & -0.620 \\
\hline & 5 & 0.2081 & 0.493 & 0.0385 & 0.2505 & 0.833 & -0.666 \\
\hline & 6 & 0.1771 & 0.506 & 0.0400 & 0.2168 & 0.844 & -0.702 \\
\hline & 10 & 0.1130 & 0.534 & 0.0432 & 0.1438 & 0.865 & -0.784 \\
\hline \multirow[t]{6}{*}{5} & 2 & 0.3654 & 0.496 & 0.0757 & 0.4266 & 0.836 & -1.36 \\
\hline & 3 & 0.2404 & 0.525 & 0.0839 & 0.2985 & 0.862 & -1.54 \\
\hline & 4 & 0.1834 & 0.527 & 0.0919 & 0.2373 & 0.881 & -1.70 \\
\hline & 5 & 0.1501 & 0.544 & 0.0971 & 0.2000 & 0.892 & -1.82 \\
\hline & 6 & 0.1279 & 0.551 & 0.1012 & 0.1743 & 0.899 & -1.92 \\
\hline & 10 & 0.0823 & 0.579 & 0.1092 & 0.1185 & 0.913 & -2.15 \\
\hline
\end{tabular}

\section{THE ORDER OF THE $I-N$ TRANSITION}

It could be argued that the order of the transition might change if we consider two or more order parameters instead of the OOP approximation. To prove that this is not the case we are going now to derive the same results as above from the exact Euler-Lagrange equation.
This equation is obtained by imposing $h(\mathbf{u})$ to be a minimum of (2.16) and keeping the normalization, $\int d \mathbf{u} h(\mathbf{u})=1$. Thus

$$
h(\mathbf{u})=\frac{\exp \left[-2 Q_{\mathrm{HS}}(\eta) \int d \mathbf{u}^{\prime} \Sigma\left(\mathbf{u} \cdot \mathbf{u}^{\prime}\right) h\left(\mathbf{u}^{\prime}\right)\right]}{\int d \mathbf{u} \exp \left[-2 Q_{\mathrm{HS}}(\eta) \int d \mathbf{u}^{\prime} \Sigma\left(\mathbf{u} \cdot \mathbf{u}^{\prime}\right) h\left(\mathbf{u}^{\prime}\right)\right]} .
$$

TABLE III. Data for the coexistence of the isotropic and the nematic phases of a system of $D$ dimensional HE's with aspect ratio $\kappa$, as obtained from the $r$-GELA. Notation is the same as in Table I except that in this case the order parameter is denoted by $q_{D}$. Again the CS is used as HS EOS.

\begin{tabular}{|c|c|c|c|c|c|c|}
\hline$D$ & $\kappa$ & $\eta_{i}$ & $\Delta \eta$ & $P^{*}$ & $\mu_{\mathrm{ex}}^{*}$ & $q_{D}$ \\
\hline \multirow[t]{6}{*}{3} & 2 & 0.693 & 0.003 & 50.1 & 88.3 & 0.477 \\
\hline & 3 & 0.523 & 0.009 & 10.7 & 28.1 & 0.536 \\
\hline & 4 & 0.424 & 0.015 & 5.21 & 17.9 & 0.588 \\
\hline & 5 & 0.358 & 0.020 & 3.37 & 14.2 & 0.627 \\
\hline & 6 & 0.312 & 0.024 & 2.49 & 12.3 & 0.657 \\
\hline & 10 & 0.208 & 0.027 & 1.24 & 9.64 & 0.709 \\
\hline \multirow[t]{6}{*}{4} & 2 & 0.503 & 0.007 & 35.4 & 95.7 & 0.729 \\
\hline & 3 & 0.346 & 0.018 & 7.94 & 35.1 & 0.767 \\
\hline & 4 & 0.266 & 0.025 & 3.85 & 23.5 & 0.797 \\
\hline & 5 & 0.217 & 0.029 & 2.46 & 19.1 & 0.820 \\
\hline & 6 & 0.183 & 0.031 & 1.79 & 16.8 & 0.837 \\
\hline & 10 & 0.115 & 0.032 & 0.86 & 13.6 & 0.875 \\
\hline \multirow[t]{6}{*}{5} & 2 & 0.369 & 0.006 & 28.8 & 107.1 & 0.661 \\
\hline & 3 & 0.241 & 0.012 & 6.94 & 43.1 & 0.715 \\
\hline & 4 & 0.182 & 0.016 & 3.47 & 29.8 & 0.754 \\
\hline & 5 & 0.148 & 0.018 & 2.26 & 24.6 & 0.782 \\
\hline & 6 & 0.125 & 0.019 & 1.66 & 21.8 & 0.802 \\
\hline & 10 & 0.079 & 0.020 & 0.82 & 17.8 & 0.846 \\
\hline
\end{tabular}


This equation has always the trivial solution $h(\mathbf{u})=1$, corresponding to the isotropic phase. To find a nonconstant solution, that would correspond to the nematic phase, we assume that (7.1) exhibits a continuous bifurcation at a point $\eta_{c}$ and apply the analysis of Kayser and Raveché [52]. This method will tell us whether indeed (7.1) has a bifurcation point and will allow us to compute it. Further, we can extend the analysis to check which kind of bifurcation we are dealing with and interpret the result in terms of the $I-N$ transition. If (7.1) has a bifurcation at a certain density, $\eta_{c}$, then at that precise point two solutions $[h(\mathbf{u})=1$ and $h(\mathbf{u}) \neq 1]$ will converge to each other. So let us express the nonconstant solution as a perturbation of the constant one, $h(\mathbf{u})=1+f(\mathbf{u})$, where $f(\mathbf{u})$ vanishes when we approach $\eta_{c}$ from above. Let us rewrite (7.1) in terms of $f(\mathbf{u})$ :

$$
f(\mathbf{u})=-1+\frac{\exp \left[-2 Q_{\mathrm{HS}}(\eta)(K f)(\mathbf{u})\right]}{\int d \mathbf{u}^{\prime} \exp \left[-2 Q_{\mathrm{HS}}(\eta)(K f)\left(\mathbf{u}^{\prime}\right)\right]}
$$

where to simplify the notation we have introduced the integral operator $K$, defined by

$$
(K f)(\mathbf{u}) \equiv \int d \mathbf{u}^{\prime} \Sigma\left(\mathbf{u} \cdot \mathbf{u}^{\prime}\right) f\left(\mathbf{u}^{\prime}\right)
$$

Since near the bifurcation $f(\mathbf{u})$ is as small as desired, we can expand the right-hand side of (7.2) as a functional of $f(\mathbf{u})$ up to first order to obtain

$$
f(\mathbf{u})=-2 Q_{\mathrm{HS}}(\eta)(K f)(\mathbf{u})+O\left(f^{2}\right) .
$$

So the existence of the bifurcation is related to the existence of eigenvalues of the operator $K$, with the restrictions that $f(\mathbf{u})=f(-\mathbf{u}), \quad \int d \mathbf{u} f(\mathbf{u})=0$, and $\mathbf{u} \cdot \mathbf{n}=$ $\cos \theta= \pm 1$ have to be the only maxima of $f(\mathbf{u})$. In that case the bifurcation is given by

$$
2 Q_{\mathrm{HS}}\left(\eta_{c}\right)=-\frac{1}{\lambda},
$$

$\lambda$ being the corresponding eigenvalue. Using the summation theorem of Gegenbauer polynomials we can prove, after some algebra, that $M_{n}^{(D)}$ are eigenfunctions of $K$ with eigenvalue $\lambda_{n}^{(D)}$ :

$$
\lambda_{n}^{(D)}=\int d \mathbf{u} \Sigma(\mathbf{u} \cdot \mathbf{n}) M_{n}^{(D)}(\mathbf{u}) .
$$

The parity condition $[f(\mathbf{u})=f(-\mathbf{u})]$ discards the odd elements of the polynomial set, the condition $\int d \mathbf{u} f(\mathbf{u})=0$ discards $\boldsymbol{M}_{0}^{(D)}(\mathbf{u})$, so finally, the $\boldsymbol{M}_{2 n}^{(D)}(\mathbf{u})$ with $n>0$ form a basis for $f(\mathbf{u})$. Further, the restriction that $f( \pm 1)$ be the unique maxima of $f(\cos \theta)$ leaves only $M_{2}^{(D)}(\mathbf{u})$ in the vicinity of $\eta_{c}$. Then, Eq. (7.5) becomes, after inserting $\lambda_{2}^{(D)}$, identical to the condition of marginal stability of the isotropic phase we found within the OOP approximation [cf. Eq. (6.2)], so that $\eta_{c}=\eta_{2}$. This is not surprising if we take into account the shape we chose for $h(\mathbf{u})$ in the OOP approximation [Eq. (3.2)]. Furthermore, this derivation of a basis of polynomials for $f(\mathbf{u})$ is the very justification of the choice we made in Sec. III.

We can go ahead with Kayser and Raveché's formalism and expand $f(\mathbf{u})$ and $r(\eta) \equiv 2 Q_{\mathrm{HS}}(\eta)$ around $\eta_{c}$ :

$$
\begin{aligned}
& f(\mathbf{u})=f_{1}(\mathbf{u}) \alpha+f_{2}(\mathbf{u}) \alpha^{2} / 2 !+\cdots, \\
& r=r_{c}+r_{1} \alpha+r_{2} \alpha^{2} / 2 !+\cdots
\end{aligned}
$$

where $\alpha$ is a parameter that measures the "distance" to the bifurcation point and which has to be eliminated to find $f(\mathbf{u})$ as a function of $\eta$. By inserting (7.7) and (7.8) in (7.2) the following hierarchy is found:

$$
\begin{aligned}
& -r_{c}\left(K f_{1}\right)(\mathbf{u})=f_{1}(\mathbf{u})-\int d \mathbf{u}^{\prime} f_{1}\left(\mathbf{u}^{\prime}\right), \\
& -r_{c}\left(K f_{2}\right)(\mathbf{u})-2 r_{1}\left(K f_{1}\right)(\mathbf{u}) \\
& =f_{2}(\mathbf{u})-\left[f_{1}(\mathbf{u})\right]^{2}-\int d \mathbf{u}^{\prime}\left\{f_{2}\left(\mathbf{u}^{\prime}\right)-\left[f_{1}\left(\mathbf{u}^{\prime}\right)\right]^{2}\right\}
\end{aligned}
$$

which can be solved by noticing that $\int d \mathbf{u}^{\prime} f_{n}\left(\mathbf{u}^{\prime}\right)=0$ and $f_{1}(\mathbf{u})=M_{2}^{(D)}(\mathbf{u})$, and multiplying the equations by $f_{1}(\mathbf{u})$ and integrating over $\mathbf{u}$, using the self-adjointness of $K$ and imposing orthogonality of the $f_{n}(\mathbf{u})$. In this way we find the following solutions up to second order:

$$
\begin{aligned}
& f_{1}(\mathbf{u})=M_{2}^{(D)}(\mathbf{u}) \\
& f_{2}(\mathbf{u})=\frac{D^{2}(D+1)}{(D+4)(D+2)(D-1)} \frac{\lambda_{2}^{(D)}}{\left(\lambda_{2}^{(D)}-\lambda_{4}^{(D)}\right)} M_{4}^{(D)}(\mathbf{u}) \\
& r_{1}=\frac{2(D-2)}{(D+4)(D-1)} \frac{1}{\lambda_{2}^{(D)}}, \\
& r_{2}=\left[\begin{array}{l}
\left(5 D^{2}-13 D+12\right) \lambda_{4}^{(D)} \\
\left.\quad-\frac{2\left(D^{4}+7 D^{3}-13 D^{2}-16 D^{2}+48\right)}{(D+4)(D+2)} \lambda_{2}^{(D)}\right) \\
\quad \times \frac{4}{(D+6)(D+4)(D-1)^{2} \lambda_{2}^{(D)}\left(\lambda_{2}^{(D)}-\lambda_{4}^{(D)}\right)}
\end{array}\right.
\end{aligned}
$$

Having in mind that $\lambda_{2}^{(D)}<0$ (otherwise the isotropic phase would always be unstable) and that $Q_{\mathrm{HS}}(\eta)$ is a monotonically increasing function of $\eta$, from (7.13) we see that $r_{1}<0$ for $D>2$, and so there exists a nonconstant solution of (7.2) for densities below $\eta_{c}$. The transition is, then, first order, in agreement with what we have deduced from the OOP approximation. On the other hand $r_{1}$ vanishes for $D=2$ so we must resort to $r_{2}$ to decide the order of the transition. Setting $D=2$ in (7.14) we obtain

$$
r_{2}=\frac{2 \lambda_{4}^{(2)}-\lambda_{2}^{(2)}}{4 \lambda_{2}^{(2)}\left(\lambda_{2}^{(2)}-\lambda_{4}^{(2)}\right)} .
$$

As it can be checked numerically that $r_{2}>0$, so no solution other than the trivial one exists for densities below $\eta_{c}$, and therefore the transition is second order for $D=2$. We see thus that the conclusions we obtained from the OOP approximation have not been affected by the approximation itself; only the location of the coexisting 
densities of the first-order transition and the results for the thermodynamic quantities may be expected to be altered by the OOP approximation.

\section{THE ONSAGER LIMIT AND THE NEMATIC PHASE OF D-DIMENSIONAL HARD SPHEROCYLINDERS}

In his first attempt to describe the $I-N$ transition, Onsager proved [9] that in 3D the virial expansion up to second order becomes exact in the combined, so-called
Onsager limit, $\eta \rightarrow 0, \kappa \rightarrow \infty$ while keeping $\kappa \eta=$ const. We can check whether our formalism is consistent with Onsager's theory by performing this limit on our functional. First, we have to take into account that the compressibility factor, in the limit $\eta \rightarrow 0$, behaves as $Z_{\mathrm{HS}}(\eta)=1+2^{D-1} \eta+O\left(\eta^{2}\right)$, which implies $Q_{\mathrm{HS}}(\eta)$ $=2^{D-1} \eta+O\left(\eta^{2}\right)$. Second, in the limit $\kappa \rightarrow \infty$ we have $\left(1-\chi^{2}\right)^{-1 / 2} \sim \kappa / 2+O(1 / \kappa)$. Combining the two expressions and defining the Onsager variable $c=B_{2} \rho \sim 2^{D-3} \pi \kappa \eta /(D-1) N_{D}^{2}$, Eq. (3.13) reduces to

$$
\begin{aligned}
\beta \phi_{\mathrm{ex}}^{\mathrm{Ons}}(\gamma ; c)=\frac{c}{\pi N_{D+1}} \int_{0}^{1} d s \int_{0}^{1} d s^{\prime} \int_{-1}^{1} d x[ & {\left[\left(1-s^{2}\right)\left(1-s^{\prime 2}\right)\right]^{(D-3) / 2} \zeta_{D}(x) } \\
& \times h_{D}(s) h_{D}\left(s^{\prime}\right)\left\{1-\left[s s^{\prime}+\left(1-s^{2}\right)^{1 / 2}\left(1-s^{\prime 2}\right)^{1 / 2} \cos (\pi x)\right]^{2}\right\}^{1 / 2}
\end{aligned}
$$

with $\zeta_{D}(x)$ given by (3.10). Equivalently, once we have found $\gamma^{*}(c)$ as the minimum of $\phi^{\mathrm{Ons}}(\gamma ; c)$ with respect to $\gamma$ for any given $c$, the compressibility factor gets the simple expression:

$$
Z_{\text {Ons }}(c)=1+\beta \phi_{\mathrm{ex}}^{\text {Ons }}\left(\gamma^{*}(c) ; c\right) .
$$

This pair of expressions, (8.1) and (8.2), coincides with those found for $D=2$ and 3 in Refs. [28] and [27], respectively. For $D=3$ they also coincide with the exact Onsager expressions. However, in spite of its oblate-prolate symmetry, the latter equations describe only approximately the system of 3D hard platelets [10], and similarly for the $2 \mathrm{D}$ hard needles system $[11,28]$, since in both cases the virial expansions cannot be truncated at second order.

An interesting consequence of the Onsager limit arises by noticing the similarity between the excluded volume part of the right-hand side of (8.1) and the excluded volume of a $D$-dimensional HSC [Eq. (4.6)]. If we work out the expressions for the excess free energy and the pressure of this system we will find the remarkable relationships

$$
\begin{aligned}
& \beta \phi_{\mathrm{ex}}^{\mathrm{HSC}}(\eta)=Q_{\mathrm{HS}}(\eta)+Z_{\mathrm{Ons}}(\bar{c}(\eta))-1, \\
& Z_{\mathrm{HSC}}(\eta)=Z_{\mathrm{HS}}(\eta)+\frac{Z_{\mathrm{HS}}(\eta)-1}{Q_{\mathrm{HS}}(\eta)}\left[Z_{\mathrm{Ons}}(\bar{c}(\eta))-1\right]
\end{aligned}
$$

TABLE IV. Data for the coexistence of the isotropic and the nematic phases of a system of 3D HSC's with aspect ratio $\kappa=6$, as obtained by molecular-dynamics (MD) simulations [13], by numerical solutions of (2.16) as reported by Lee [30], from the functional (2.14) as given by Holyst and Poniewierski [26], and from the present calculations. Notation is the same as in Table I and the HS EOS is also the CS.

\begin{tabular}{lllll}
\hline \hline & $\eta_{i}$ & $\Delta \eta$ & $P^{*}$ & $q$ \\
\hline MD & 0.4 & & 4.9 & \\
Ref. [30] & 0.400 & 0.018 & 5.36 & 0.67 \\
Ref. [26] & 0.38 & 0.03 & 2.9 & 0.59 \\
$r$-GELA & 0.401 & 0.017 & 5.42 & 0.67 \\
$t$-GELA & 0.315 & 0.039 & 2.5 & 0.71 \\
\hline \hline
\end{tabular}

where the effective density $\bar{c}(\eta)$ is given by

$$
\bar{c}(\eta)=\frac{D N_{D+1}}{2 \pi N_{D}} \frac{(\kappa-1)^{2}}{\left[1+(\kappa-1) / N_{D+2}\right]} Q_{\mathrm{HS}}(\eta) .
$$

From these equations, almost all features of the isotropic and nematic phases of finite $\kappa$ HSC can be obtained through the knowledge of those of the hard needles system (Onsager limit). Thus the order parameter is simply given by $q_{D}^{\mathrm{HSC}}(\eta)=q_{D}^{\text {Ons }}(\bar{c}(\eta))$; the thermodynamics is computed with the aid of (8.3)-(8.5), and the stability limit of both phases $\left(\eta_{0}\right.$ and $\left.\eta_{2}\right)$ together with the point of marginal stability $\left(\eta_{1}\right)$ can be obtained from the relation $\bar{c}\left(\eta_{j}\right)=c_{j}\left(j=0,1,2\right.$ and $c_{j}$ being the equivalent quantities in the Onsager theory. However, since Eqs. (8.3) and (8.4) are written in terms of both variables, $\eta$ and $\bar{c}$, the coexistence has to be solved for each $\kappa$ independently. In Table IV results for the coexistence in the 3D HSC system with $\kappa=6$ are gathered together with the available simulation results [13], the results from the $r$-GELA with numerical determination of $h(\mathbf{u})$ by Lee [30], the results from the $t$-GELA by Holyst and Poniewierski [26], and the present calculations with both approaches. The superiority of the $r$-GELA compared to the other theories can again be appreciated. Besides, it can be seen that the numerical solution of the Euler-Lagrange equation [30] does not lead to any serious improvement and this makes the OOP approximation a convenient and simple approximation.

\section{THE LIMIT $D \rightarrow \infty$}

The study of first-order phase transitions in the large- $D$ limit is interesting due to the fact that in this limit the mean-field theory becomes exact, unlike the case of the second-order transitions for which the mean-field approach is exact above a certain finite (critical) dimension. The former statement can be understood by noticing that the average number of neighbors goes to infinity with the dimension, satisfying in this way the assumptions of the mean-field theory [53]. The latter statement is a wellknown consequence of the renormalization group. This large- $D$ study has been recently applied to some classical 
problems in condensed matter, namely, the freezing of HS [54], the $I-N$ transition of hard rods [55], and the phase diagram of binary HS mixtures [56]. In the remainder of this section we will extend the study of the $I-N$ transition of hard rods [55] to the case of HE's in the GOA, showing that our formalism in the OOP approximation is exact in this limit.

First of all we have to define a new density variable which remains finite when the dimension goes to infinity. Let us introduce $\xi \equiv 2^{D} H_{D}^{0} \eta / D$. In the $D \rightarrow \infty$ limit the free-energy virial expansion truncates at second order, so that Onsager's functional becomes exact [see Eqs. (2.14) and (2.16) and comments below]. Let us further assume that the angular probability function $h(\theta) \sin ^{D-2} \theta$, i.e., the probability of finding a molecule with orientation between $\theta$ and $\theta+d \theta$, is very peaked around a certain value $\theta_{0}$. Then, an asymptotic expansion of the integrals involved leads to

$$
h(\theta)=R \exp \left[-D \xi\left(1-\chi^{2} \cos ^{2} \theta_{0} \cos ^{2} \theta\right)^{1 / 2}\right]
$$

with $R$ the normalization factor. By imposing $\theta_{0}$ to be the maximum of the angular probability function we obtain

$$
\begin{aligned}
& u=0, \quad \xi<\xi_{m}, \\
& \xi^{2}=\frac{1-\chi^{2} u^{2}}{\chi^{4} u^{2}(1-u)^{2}}, \quad \xi>\xi_{m},
\end{aligned}
$$

where $u \equiv \cos ^{2} \theta_{0}$ and $\xi_{m}^{2} \equiv\left(1-\chi^{2} u_{m}^{2}\right) / \chi^{4} u_{m}^{2}\left(1-u_{m}\right)^{2}$, with $u_{m}$ the solution of $\chi^{2} u_{m}^{3}-2 u_{m}+1=0$ satisfying $0<u_{m}<1$. On the other hand, for large $D$ we obtain asymptotically:

$$
\begin{array}{rl}
\ln R=-\ln \int_{0}^{\pi / 2} & d \theta \frac{1}{N_{D}} \\
& \times \exp \left[-D \xi\left(1-\chi^{2} \cos ^{2} \theta_{0} \cos ^{2} \theta\right)^{1 / 2}\right] \\
& \times \sin ^{D-2} \theta \\
\sim & D\left[\xi\left(1-\chi^{2} \cos ^{4} \theta_{0}\right)^{1 / 2}-\ln \left(\sin \theta_{0}\right)\right]
\end{array}
$$

so that for $\xi>\xi_{m}$, i.e., in the nematic phase

$$
\ln R \sim \frac{1-\chi^{2} u^{2}}{\chi^{2} u(1-u)}-\frac{1}{2} \ln (1-u)
$$

The excess free energy behaves in this limit as $\beta \phi_{\mathrm{ex}} \sim D\left(1-\chi^{2} u^{2}\right)^{1 / 2} \xi / 2$, the final expression for the free energy of the nematic fluid being

$$
\beta \phi_{n} \sim \frac{D}{2}\left[\frac{1-\chi^{2} u^{2}}{\chi^{2} u(1-u)}-\ln (1-u)\right]+c_{D}
$$

with $c_{D} \sim D \ln \left(\Lambda^{1 / D} / 2\right)$ the ideal-gas contribution. The pressure can be obtained through the thermodynamic relation $\beta P / \rho=\xi \partial(\beta \phi) / \partial \xi$ yielding

$$
\beta \frac{P_{n}}{\rho} \sim \frac{D}{2} \frac{1-\chi^{2} u^{2}}{\chi^{2} u(1-u)}
$$

and then from (2.18) the chemical potential reads

$$
\beta \mu_{n} \sim D\left[\frac{1-\chi^{2} u^{2}}{\chi^{2} u(1-u)}-\frac{1}{2} \ln (1-u)\right]+c_{D} .
$$

Finally, if $\xi<\xi_{m}$, i.e., in the isotropic phase:

$$
\begin{aligned}
& \beta \phi_{i} \sim D \frac{\xi}{2}+c_{D}, \\
& \beta \frac{P_{i}}{\rho} \sim D \frac{\xi}{2}, \\
& \beta \mu_{i} \sim D \xi+c_{D} .
\end{aligned}
$$

Now let us go back to our formalism and take the expressions deduced in Sec. III within the OOP approximation. In the $D \rightarrow \infty$ limit the polynomial basis (3.1) becomes [39] $M_{n}^{(\infty)}(x)=x^{n}$, and Eqs. (3.2) and (3.3) reduce to

$$
\begin{aligned}
& h_{D}(\theta) \sim \Omega_{D}(\gamma)^{-1} \exp \left(\gamma \cos ^{2} \theta\right), \\
& \ln \Omega_{D}(\gamma) \sim \ln \int_{0}^{\pi / 2} d \theta \sin ^{D-2} \theta \exp \left(\gamma \cos ^{2} \theta\right),
\end{aligned}
$$

where we have used the fact that $\ln N_{D}=O(\ln D)$ and can thus be dropped. If the angular probability distribution has to be peaked around a certain angle $\theta_{0}$, then $\gamma$ has to increase linearly with $D$ for large $D$, i.e., $\gamma \sim D g$. With this assumption Eq. (9.12) can be rewritten as

$$
\ln \Omega_{D} \sim \ln \int_{0}^{\pi / 2} d \theta \exp \left\{D\left[g \cos ^{2} \theta+\ln (\sin \theta)\right]\right\} .
$$

The dominant contribution to this integral comes from the maximum of the argument in the exponential, $y(\theta) \equiv g \cos ^{2} \theta+\ln (\sin \theta)$, i.e., the angle $\theta_{0}$ such that $y^{\prime}\left(\theta_{0}\right)=0$. This condition leads to the equation $\cot \theta_{0}=g \sin \left(2 \theta_{0}\right)$, whose solutions are

$$
\begin{aligned}
& \cos \theta_{0}=0, \quad 2 g<1, \\
& 2 g \sin ^{2} \theta_{0}=1, \quad 2 g>1 .
\end{aligned}
$$

The above result allows us to compute (9.13), obtaining

$$
\ln \Omega_{D} \sim\left\{\begin{array}{l}
0, \quad 2 g<1 \\
\frac{D}{2}[2 g-1-\ln (2 g)], \quad 2 g>1 .
\end{array}\right.
$$

On the other hand, the order parameter $q_{D}$ can be computed from (3.8) as

$$
\begin{aligned}
q \equiv q_{\infty} & =\frac{\partial}{\partial g}\left(\lim _{D \rightarrow \infty} \frac{1}{D} \ln \Omega_{D}(D g)\right) \\
& =\left\{\begin{array}{l}
0, \quad 2 g<1 \\
1-\frac{1}{2 g}, \quad 2 g>1 .
\end{array}\right.
\end{aligned}
$$

Using this relation together with Eq. (9.15) into (3.11) the following expression for the ideal contribution to the free energy is found:

$$
\beta \phi_{\mathrm{id}}=-\frac{D}{2} \ln (1-q)+c_{D} .
$$


Notice that this formula is valid for all $g$. To compute the excess free-energy contribution we have to asymptotically expand (3.9) for large $D$. Because the angular probability distribution is sharply peaked we get

$$
H_{D} \sim\left(\frac{1-\chi^{2} q^{2}}{1-\chi^{2}}\right)^{1 / 2}
$$

Besides, $Q_{\mathrm{HS}}(\eta) \sim D \xi / 2 H_{D}^{0}$, so that both the $t$-GELA and the $r$-GELA yield the same expression for the excess free energy, that is,

$$
\beta \phi_{\mathrm{ex}} \sim \frac{D}{2} \xi\left(1-\chi^{2} q^{2}\right)^{1 / 2}
$$

Thus the free energy within the present formalism reaches the final form:

$$
\beta \phi \sim \frac{D}{2}\left[-\ln (1-q)+\xi\left(1-\chi^{2} q^{2}\right)^{1 / 2}\right]+c_{D} .
$$

Once we have obtained the (Landau) expression for the free energy as a function of the order parameter $q$, we just have to find the $q$ value that minimizes it to determine where the nematic phase is stable. Doing this leads to the following relation:

$$
\xi^{2}=\frac{1-\chi^{2} q^{2}}{\chi^{4} q^{2}(1-q)^{2}} .
$$

Comparison of this formula with Eq. (9.2) yields the identification $q=u$ and the conclusion that $q(\xi)$ obtained by the present method is exact. Further, we can use (9.21) to eliminate $\xi$ in (9.20) and obtain the free energy of the nematic phase:

$$
\beta \phi_{n} \sim \frac{D}{2}\left[\frac{1-\chi^{2} q^{2}}{\chi^{2} q(1-q)}-\ln (1-q)\right]+c_{D},
$$

which with the previous identification of the order parameter turns out to be exactly the same as the free energy (9.5). Therefore the present formalism within the OOP approximation becomes exact in the large- $D$ limit, whatever the version (either $t$-GELA or $r$-GELA) we use (this is reminiscent of the fact that both expressions coincide up to second order in the virial expansion). The expressions for the pressure and the chemical potential and those for the isotropic phase are consequently identical to the exact ones. In Fig. 9 the EOS for $\infty$-dimensional HE's is plotted versus the variable $\xi$ for several values of the aspect ratio. The isotropic branch is independent of $\kappa$ [see (9.9)]. The nematic branches are joined to the isotropic branch by a horizontal line that represents the tie line. As usual, this coexistence is determined by requiring both phases to have the same pressure and chemical potential. The coexisting densities are given by

$$
\begin{aligned}
& \xi_{i}=\frac{\left(1-\chi^{2} q_{c}^{2}\right)^{3 / 4}}{\chi^{2} q_{c}\left(1-q_{c}\right)}, \\
& \xi_{n}=\frac{\left(1-\chi^{2} q_{c}^{2}\right)^{1 / 2}}{\chi^{2} q_{c}\left(1-q_{c}\right)},
\end{aligned}
$$

where $q_{c}$ is the order parameter at $\xi_{n}$ and is determined

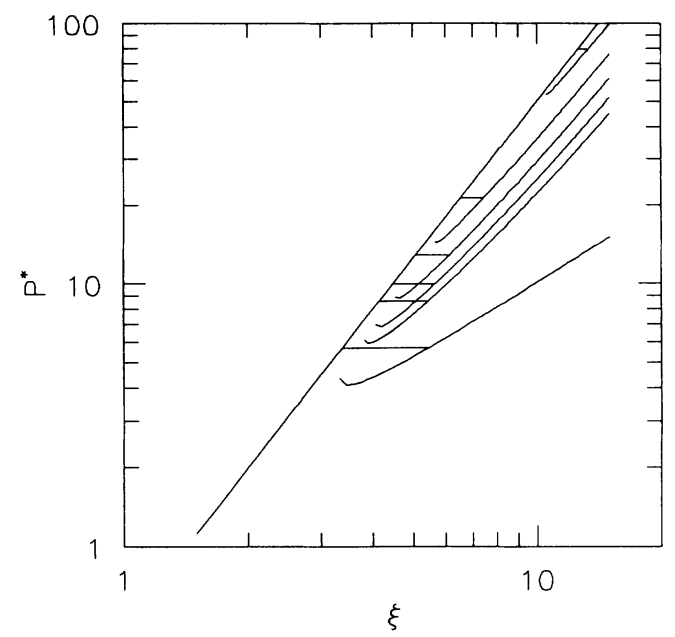

FIG. 9. Reduced pressure $P^{*}=\xi \beta P / D \rho$ vs the rescaled density $\xi=\rho 2^{D} v_{0} / D\left(1-\chi^{2}\right)^{1 / 2}$, with $\chi=\left(k^{2}-1\right) /\left(k^{2}+1\right)$, of a system of $D$-dimensional HE's in the GOA, when $D \rightarrow \infty$. The isotropic branch is represented by a straight line which is independent of $\kappa$. The nematic branch is shown for $\kappa=2,3,4,5,6$, and $\infty$ (from top to bottom). The tie line (also shown) indicates clearly how the isotropic-nematic transition is pushed to higher densities and also becomes narrower when $\kappa$ decreases from the original Onsager limit $(\kappa=\infty)$ to the spherical limit $(\kappa=1)$ where the transition disappears.

by the equation

$\left(1-\chi^{2} q_{c}^{2}\right)^{3 / 4}=1-\chi^{2} q_{c}^{2}-\frac{\chi^{2}}{2} q_{c}\left(1-q_{c}\right) \ln \left(1-q_{c}\right)$.

In Fig. 10 the order parameter $q$ is represented as a function of $\xi$ for several aspect ratios. Also in the same figure

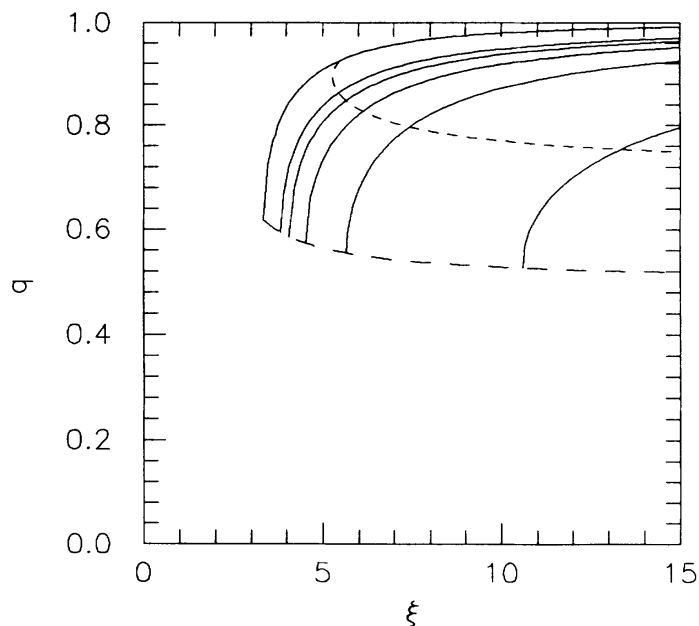

FIG. 10. Order parameter $q$ as a function of the rescaled density $\boldsymbol{\xi}$ (see caption of Fig. 9) for a system of $D$-dimensional HE's in the GOA, when $D \rightarrow \infty$, for a set of aspect ratios $\kappa=2,3,4,5$, 6 , and $\infty$ (solid lines from right to left). The long-dashed line represents the order parameter at the density $\xi_{m}$ where the nematic phase appears for the first time as a metastable phase (when increasing $\xi$ ). The short-dashed line represents the order parameter at the density $\xi_{n}$ at which the nematic phase coexists with the isotropic phase. 
$q_{c}\left(\xi_{n}\right)$ and $q_{m} \equiv u_{m}\left(\xi_{m}\right)$ are plotted, $\xi_{m}$ [appearing in (9.2)] being the lower stability limit of the nematic phase. Notice the huge jump in the order parameter at the $I-N$ transition, indicating a strong first-order transition.

\section{CONCLUSIONS}

We have shown how the ELA can be applied to study the $I-N$ transition of HCB in any dimension $D$. The results can at present only be tested for $D=2,3$, and $\infty$. For $D=3$ our results coincide with those of the functional proposed by Lee [30], and turn out to provide the best description presently available. The great advantage of our formalism, however, is that it gives a derivation of this functional based upon the exact DFT relations and thus all the approximations involved in the final expression are made clear. In particular we can identify Pynn's approximation to the DCF as being responsible for the factorization of the radial and angular parts of the functional. This factorization is a good approximation in $3 \mathrm{D}$ and presumably also for higher dimensions (because the results are proven to become exact in the large- $D$ limit), but leads to a failure of the theory in $2 \mathrm{D}$, where a second-order transition is predicted while simulations show a more complex behavior (first-order becoming continuous - of the Kosterlitz-Thouless type - for higher eccentricities). Furthermore, the isotropic EOS predicted in $2 \mathrm{D}$ is incorrect since the factorization induces a rescaling of all virial coefficients by the second one [see Eq. (5.2)], which vanishes in the large eccentricity limit as $\kappa^{-1}$. This implies that the rescaled virial coefficients vanish in this limit as $b_{n} \sim \kappa^{-(n-1)}$, instead of going to a nonzero value, as the simulations indicate [11]. Besides, the OOP approximation does not alter the nature of the transition and only slightly changes the numerical results. For these reasons we conjecture that an improvement of the underlying DCF is needed in order to obtain reasonable results for a $2 \mathrm{D}$ system.

\section{ACKNOWLEDGMENTS}

We are grateful to Hong $\mathrm{Xu}$ for allowing us to use some of her unpublished material on hard spherocylinders. One of us (J.A.C.) is also indebted to her for some very valuable discussions. We are also grateful to Patricia Lamas. This work has been partially supported by a grant from the Dirección General de Investigación Científica y Técnica (Spain) under Grant No. PB88-0140. One of us (M.B.) acknowledges the financial support of the Fonds National de la Recherche Scientifique and also from the Association Euratom-Etat Belge.

\section{APPENDIX}

Let us introduce the following notation:

$\Psi(\widehat{\rho} ;[\rho]) \equiv-\frac{1}{V \rho} \int d \mathbf{x} \int d \mathbf{x}^{\prime} \rho(\mathbf{x}) \rho\left(\mathbf{x}^{\prime}\right) c\left(\mathbf{r}-\mathbf{r}^{\prime} ; \mathbf{u}, \mathbf{u}^{\prime} ; \hat{\rho}\right)$

$\psi(\rho) \equiv-\rho \int_{0}^{1} d \lambda(1-\lambda) \int d \mathbf{u} \int d \mathbf{u}^{\prime} \int d \mathbf{r} c\left(\mathbf{r} ; \mathbf{u}, \mathbf{u}^{\prime} ; \lambda \rho\right)$.
The GELA amounts to computing the excess free energy as [Eq. (3.15a) of Ref. [7] ]

$$
\beta \phi_{\mathrm{ex}}[\rho]=\psi(\bar{\rho}[\rho])
$$

with $\bar{\rho}[\rho]$ defined by [Eq. (3.14) of Ref. [7] ]

$$
\frac{\partial^{2}}{\partial \lambda^{2}}[\lambda \beta \phi(\bar{\rho}[\lambda \rho])]=\Psi(\bar{\rho}[\lambda \rho] ;[\rho]) \text {. }
$$

For a given $\rho(\mathbf{x}), \bar{\rho}[\lambda \rho]$ is a function of $\lambda$, so we can define

$$
\begin{aligned}
& x(\lambda) \equiv \bar{\rho}[\lambda \rho], \\
& y(\lambda) \equiv \frac{\partial}{\partial \lambda}[\lambda \psi(x)] .
\end{aligned}
$$

For $\lambda=0$ we get $x(0)=\bar{\rho}[0]=0$ and if we expand (A6)

$$
y(\lambda)=\psi(x)+\lambda \psi^{\prime}(x) x^{\prime}(\lambda)
$$

(the prime denotes a derivative with respect to the corresponding argument) and further impose regularity at $\lambda=0$, i.e., $\left|x^{\prime}(0)\right|<\infty$ [a condition related to the existence of the expansion (3.31) of Ref. [7]], then we will have $y(0)=0$ as well. Equations (A4) and (A7) yield the following system of ordinary differential equations:

$$
\begin{aligned}
& \frac{d x}{d \lambda}=\frac{y-\psi(x)}{\lambda \psi^{\prime}(x)}, \\
& \frac{d y}{d \lambda}=\Psi(x ;[\rho])
\end{aligned}
$$

with initial conditions $x(0)=y(0)=0$, whose solution, $x(\lambda)$ allows us to write (A3) as

$$
\beta \phi_{\mathrm{ex}}[\rho]=\psi(x(1)) .
$$

Note that at $\lambda=0$ we can replace the right-hand side of (A8) by its limit when $\lambda \rightarrow 0$ :

$$
x^{\prime}(0)=\lim _{\lambda \rightarrow 0} \frac{y-\psi(x)}{\lambda \psi^{\prime}(x)}=\lim _{\lambda \rightarrow 0} \frac{y^{\prime}-\psi^{\prime}(x) x^{\prime}}{\psi^{\prime}(x)+\lambda \psi^{\prime \prime} x^{\prime}} .
$$

The derivatives of $\psi(\rho)$ can be obtained from the thermodynamics relations:

$\rho \psi^{\prime}(\rho)=\rho \frac{\partial}{\partial \rho}\left[\beta \phi_{\mathrm{ex}}(\rho)\right]=\beta \frac{P(\rho)}{\rho}-1=\sum_{n=1}^{\infty} B_{n+1} \rho^{n}$

with $P(\rho)$ the pressure of the fluid and $B_{n}, n \geq 2$, the virial coefficients. Thus $\psi^{\prime}(0)=B_{2}$ and $\psi^{\prime \prime}(0)=B_{3}$, and (A11) finally reads

$$
2 B_{2} x^{\prime}(0)=y^{\prime}(0)=\Psi(0 ;[\rho]) .
$$

In the case of the $I-N$ transition, $x(\lambda)=\bar{\eta}[\lambda \rho]$ and $y(\lambda)$ is redefined consequently. Working out the expressions as in Sec. II, the system (A8) and (A9) becomes

$$
\frac{d x}{d \lambda}=\frac{x}{\lambda}\left[\frac{y-Q_{\mathrm{HS}}(x)}{Z_{\mathrm{HS}}(x)-1}\right),
$$




$$
\frac{d y}{d \lambda}=\eta_{s}[h] \frac{d}{d x}\left[Z_{\mathrm{HS}}(x)-1+Q_{\mathrm{HS}}(x)\right],
$$

where $\eta_{s}[h]$ depends on whether we are imposing the self-consistency on the whole excess free energy, in which case we obtain

$$
\eta_{s}[h] \equiv \eta \frac{\int d \mathbf{u} \int d \mathbf{u}^{\prime} h(\mathbf{u}) h\left(\mathbf{u}^{\prime}\right) \Sigma\left(\mathbf{u} \cdot \mathbf{u}^{\prime}\right)}{\int d \mathbf{u} \int d \mathbf{u}^{\prime} \Sigma\left(\mathbf{u} \cdot \mathbf{u}^{\prime}\right)}
$$

or just on the radial part, in which case we simply have $\eta_{s}[h]=\eta$. It can be easily checked that the solution to (A8) and (A9) with $x(0)=y(0)=0$ is $x(\lambda)=\lambda \eta_{s}[h]$.
[1] B. J. Alder and T. E. Wainwright, J. Chem. Phys. 27, 1208 (1957).

[2] B. J. Alder and T. E. Wainwright, Phys. Rev. 127, 359 (1962).

[3] See M. Baus, J. Stat. Phys. 48, 1129 (1987); and J. Phys. Condens. Matter 2, 2111 (1990) for a review of the DFT applied to the study of freezing.

[4] R. Evans, Adv. Phys. 28, 143 (1979).

[5] T. V. Ramakrishnan and M. Yussouf, Phys. Rev. B 19, 2775 (1979).

[6] J. P. Hansen and I. R. McDonald, Theory of Simple Liquids (Academic, London, 1986).

[7] J. F. Lutsko and M. Baus, Phys. Rev. A 41, 6647 (1990).

[8] P. G. de Gennes, The Physics of Liquid Crystals (Clarendon, Oxford, 1974).

[9] L. Onsager, Ann. N. Y. Acad. Sci. 51, 627 (1949).

[10] R. Eppenga and D. Frenkel, Mol. Phys. 52, 1303 (1984).

[11] D. Frenkel and R. Eppenga, Phys. Rev. A 31, 1776 (1985).

[12] A. Stroobants, H. N. W. Lekkerkerker, and D. Frenkel, Phys. Rev. A 36, 2929 (1987).

[13] D. Frenkel, J. Phys. Chem. 92, 3280 (1988); J. A. C. Veerman and D. Frenkel, Phys. Rev. A 41, 3237 (1990).

[14] M. P. Allen and M. R. Wilson, J. Comput.-Aided Mol. Design 3, 335 (1989).

[15] D. Frenkel and B. M. Mulder, Mol. Phys. 55, 1171 (1985).

[16] M. P. Allen, Liq. Cryst. 8, 499 (1990).

[17] J. Vieillard-Baron, J. Chem. Phys. 56, 4729 (1972).

[18] J. A. Cuesta and D. Frenkel, Phys. Rev. A 42, 2126 (1990).

[19] M. D. Lipkin and D. W. Oxtoby, J. Chem. Phys. 79, 1939 (1983); T. J. Sluckin and P. Shukla, J. Phys. A 16, 1539 (1983).

[20] B. M. Mulder and D. Frenkel, Mol. Phys. 55, 1193 (1985).

[21] B. Tjipto-Margo and G. T. Evans, J. Chem. Phys. 93, 4254 (1990).

[22] U. P. Singh and Y. Singh, Phys. Rev. A 33, 2725 (1986).

[23] J. F. Marko, Phys. Rev. Lett. 60, 325 (1988).

[24] A. Perera, G. N. Patey, and J. J. Weis, J. Chem. Phys. 89, 6941 (1988).

[25] B. M. Mulder, Phys. Rev. A 35, 3095 (1987); A. M. Somoza and P. Tarazona, J. Chem. Phys. 91, 517 (1989); R. Holyst and A. Poniewierski, Phys. Rev. A 39, 2742 (1989); A. Poniewierski and R. Holyst, ibid. 41, 6871 (1990).

[26] R. Holyst and A. Poniewierski, Mol. Phys. 68, 381 (1989).

[27] M. Baus, J. L. Colot, X. G. Wu, and H. Xu, Phys. Rev. Lett. 59, 2184 (1987); J. L. Colot, X. G. Wu, H. Xu, and M. Baus, Phys. Rev. A 38, 2022 (1988).

[28] J. A. Cuesta, C. F. Tejero, and M. Baus, Phys. Rev. A 39, 6498 (1989).

[29] J. A. Cuesta, C. F. Tejero, H. Xu, and M. Baus, Phys. Rev. A 44, 5306 (1991).

[30] S. D. Lee, J. Chem. Phys. 87, 4932 (1987); 83, 7036 (1988).

[31] L. S. Ornstein and F. Zernike, Proc. Acad. Sci. Amsterdam 17, 793 (1914).
[32] Notice that $h(\mathbf{u})$ really denotes $h(\mathbf{u} \cdot \mathbf{n})$, with $\mathbf{n}$ the nematic director. This notation will be used throughout the paper, so whenever a function of $\mathbf{u}$ appears it must be understood to be a function of the scalar $\mathbf{u} \cdot \mathbf{n}$.

[33] For the solution of a 1D fluid with nearest-neighbor interaction in an arbitrary external field see J. K. Percus, in Simple Models of Equilibrium and Nonequilibrium Phenomena, edited by J. L. Lebowitz (North-Holland, Amsterdam, 1987); the solution of a simple 1D model with anisotropic hard-core interactions is given in C. F. Tejero and J. A. Cuesta, Physica A 168, 942 (1990); solutions for 1D spin models can be found in J. K. Percus, J. Stat. Phys. 16, 299 (1977); and C. F. Tejero, ibid. 48, 531 (1987).

[34] R. Pynn, J. Chem. Phys. 60, 4579 (1974).

[35] M. Baus, J. Phys. Condens. Matter 1, 3131 (1989).

[36] M. Baus and J. L. Colot, Phys. Rev. A 36, 3912 (1987).

[37] P. Tarazona, Phys. Rev. A 31, 2672 (1985).

[38] H. N. W. Lekkerkerker, Ph. Coulon, R. Van der Haegen, and R. Debliek, J. Chem. Phys. 80, 3427 (1984).

[39] Handbook of Mathematical Functions, edited by $\mathbf{M}$. Abramowitz and I. A. Stegun (Dover, New York, 1965); Table of Integrals, Series and Products, edited by I. S. Gradshteyn and I. M. Ryzhik (Academic, New York, 1980).

[40] S. D. Lee and R. B. Meyer, J. Chem. Phys. 84, 3443 (1986).

[41] T. Boublik and I. Nezbeda, Collect. Czech. Chem. Commun. 51, 2301 (1986).

[42] B. J. Berne and P. Pechukas, J. Chem. Phys. 56, 4213 (1972).

[43] J. A. Cuesta and C. F. Tejero, Phys. Lett. A 152, 15 (1991).

[44] I. Balberg, C. H. Anderson, S. Alexander, and N. Wagner, Phys. Rev. B 30, 3933 (1984).

[45] It seems clear that the cylinders become hypercylinders and the spheres hyperspheres when we go to a $D$ dimensional space. However, generalizing the parallelepiped is not trivial. We know that the projection on an adequately chosen plane has to have the form shown in Fig. 1, so the parallelogram remains as the shape of a couple of its "sides." And the other $(D-1)$ couples of sides are all equal to the intersection of one of the hypercylinders with a hyperplane that contains the symmetry axis. If we write the equation of the hypercylinder as $x_{1}^{2}+\cdots+x_{D-1}^{2} \leq W^{2}$ and $x_{D}^{2} \leq L^{2} / 4$ and the equation of the hyperplane as $x_{1}=0$, then the intersection appears as a $(D-1)$-hypercylinder (notice that this means a segment of length $L$ for $D=2$ and a rectangle of sides $L$ and $2 W$ for $D=3$ ).

[46] Y. Song and E. A. Mason, Phys. Rev. A 41, 3121 (1990).

[47] T. Boublík, Mol. Phys. 27, 1415 (1974); 29, 421 (1975).

[48] Y. Rosenfeld, J. Chem. Phys. 89, 4272 (1988).

[49] G. Tarjus, P. Viot, S. M. Ricci, and J. Talbot, Mol. Phys. 73, 773 (1991).

[50] J. A. Cuesta and D. Frenkel (unpublished). 
[51] M. Rigby, Mol. Phys. 66, 1261 (1989); D. Frenkel, ibid. 65, 493 (1988).

[52] R. F. Kayser, Jr. and H. J. Raveché, Phys. Rev. A 17, 2067 (1978).

[53] M. E. Mann, C. H. Marshall, and A. D. J. Haymet, Mol. Phys. 66, 493 (1989).

[54] H. L. Frisch, N. Rivier, and D. Wyler, Phys. Rev. Lett.
54, 2061 (1985); H. L. Frisch and J. K. Percus, Phys. Rev. A 35, 4696 (1987).

[55] H. O. Carmesin, H. L. Frisch, and J. K. Percus, Phys. Rev. B 40, 9416 (1989).

[56] H. O. Carmesin, H. L. Frisch, and J. K. Percus, J. Stat. Phys. 63, 791 (1991). 